\title{
A Developmental Analysis of Juxtavascular Microglia Dynamics and Interactions with the Vasculature
}

\author{
Erica Mondo, ${ }^{1}$ Shannon C. Becker, ${ }^{1 *}$ Amanda G. Kautzman, ${ }^{1 *}$ Martina Schifferer, ${ }^{2}$ Christina E. Baer, ${ }^{3}$ \\ Jiapei Chen, ${ }^{4,5}$ Eric J. Huang, ${ }^{4,5}$ Mikael Simons, ${ }^{2,6,7}$ and ${ }^{\circledR}$ Dorothy P. Schafer ${ }^{1}$ \\ ${ }^{1}$ Department of Neurobiology, Brudnick Neuropsychiatric Research Institute, University of Massachusetts Medical School, Worcester, \\ Massachusetts 01605, ${ }^{2}$ German Center for Neurodegenerative Disease, 81377 Munich, Germany, ${ }^{3}$ Sanderson Center for Optical Experimentation, \\ University of Massachusetts Medical School, Worcester, Massachusetts 01605, ${ }^{4}$ Department of Pathology, University of California, San Francisco, \\ San Francisco, California 94143, ${ }^{5}$ Pathology Service (113B), San Francisco VA Medical Center, San Francisco, CA 94121, ${ }^{6}$ Institute of Neuronal Cell \\ Biology, Technical University Munich, 80802 Munich, Germany, and ${ }^{7}$ Munich Cluster of Systems Neurology (SyNergy), 81377 Munich, Germany
}

Microglia, a resident CNS macrophage, are dynamic cells, constantly extending and retracting their processes as they contact and functionally regulate neurons and other glial cells. There is far less known about microglia-vascular interactions, particularly under healthy steady-state conditions. Here, we use the male and female mouse cerebral cortex to show that a higher percentage of microglia associate with the vasculature during the first week of postnatal development compared with older ages and that the timing of these associations is dependent on the fractalkine receptor (CX3CR1). Similar developmental microglia-vascular associations were detected in the human brain. Using live imaging in mice, we found that juxtavascular microglia migrated when microglia are actively colonizing the cortex and became stationary by adulthood to occupy the same vascular space for nearly 2 months. Further, juxtavascular microglia at all ages associate with vascular areas void of astrocyte endfeet, and the developmental shift in microglial migratory behavior along vessels corresponded to when astrocyte endfeet more fully ensheath vessels. Together, our data provide a comprehensive assessment of microglia-vascular interactions. They support a mechanism by which microglia use the vasculature to migrate within the developing brain parenchyma. This migration becomes restricted on the arrival of astrocyte endfeet such that juxtavascular microglia become highly stationary and stable in the mature cortex.

Key words: astrocytes; development; microglia; neural-immune; vasculature

Significance Statement

We report the first extensive analysis of juxtavascular microglia in the healthy, developing, and adult brain. Live imaging revealed that juxtavascular microglia within the cortex are highly motile and migrate along vessels as they are colonizing cortical regions. Using confocal, expansion, super-resolution, and electron microscopy, we determined that microglia associate with the vasculature at all ages in areas lacking full astrocyte endfoot coverage and motility of juxtavascular microglia ceases as astrocyte endfeet more fully ensheath the vasculature. Our data lay the fundamental groundwork to investigate microglia-astrocyte cross talk and juxtavascular microglial function in the healthy and diseased brain. They further provide a potential mechanism by which vascular interactions facilitate microglial colonization of the brain to later regulate neural circuit development.

\section{Introduction}

While of myeloid lineage, microglia are now appreciated to be key cellular components of neural circuits. Imaging studies have

\footnotetext{
Received Dec. 18, 2019; revised June 17, 2020; accepted June 19, 2020.

Author contributions: E.J.H., M. Simons, and D.P.S. designed research; E.M., S.C.B., M. Schifferer, C.E.B., and

J.C. performed research; E.M., S.C.B., and A.G.K. analyzed data; E.M. and D.P.S. wrote the paper.

*S.C.B. and A.G.K. contributed equally to this work.

The authors declare no competing financial interests.

This work was funded by National Institute of Mental Health Grant R01-MH-113743 (to D.P.S.), National Institute of Allergy and Infectious Diseases Grant T32-A1-095213 (to E.M.), AHA Predoctoral Fellowship 19PRE3480616 (to J.C.), the Brain \& Behavior Research Foundation (D.P.S.), the Charles H. Hood Foundation (to D.P.S.), the Dr. Miriam and Sheldon G. Adelson Medical Research Foundation (D.P.S. and M. Simmons), and Deutsche Forschungsgemeinschaft (German Research Foundation) under Germany's
}

revealed that microglia are constantly extending and retracting their processes, which are in frequent contact with neurons, synapses, and other glial cells (Davalos et al., 2005; Nimmerjahn et al., 2005; Tremblay et al., 2010; Schafer et al., 2012; Frost and

Excellence Strategy within the framework of the Munich Cluster for Systems Neurology (EXC 2145 SyNergy-ID 390857198, to M. Schifferer). The human prenatal brain tissues were provided by the Pediatric Neuropathology Research Laboratory at the University of California, San Francisco, which is supported by National Institute of Neurological Disorders and Stroke Grant P01-NS-083513 (to E.J.H.). We thank Oleg Butovsky (Brigham and Women's Hospital, Harvard University) for providing the anti-P2RY12 antibody and Georg Kislinger for help with electron microscopy.

Correspondence should be addressed to Dorothy P. Schafer at Dorothy.schafer@umassmed.edu.

https://doi.org/10.1523/JNEUROSCI.3006-19.2020

Copyright $\odot 2020$ the authors 
Schafer, 2016). These descriptions of interactions between microglia and other resident CNS cell types have now led to a new understanding that microglia are important for neural circuit structure and function, including their role in developmental synaptic pruning by engulfing and removing synapses from less active neurons (Tremblay et al., 2010; Paolicelli et al., 2011; Schafer et al., 2012; Gunner et al., 2019). In addition to interactions with parenchymal neurons and glia, microglia are known to interact with the vasculature. However, the vast majority of these studies have been in the context of disease where parenchymal microglia rapidly associate with the brain vasculature following breakdown of the blood-brain barrier (BBB) and, in turn, inflammatory microglia can modulate the breakdown of the BBB (Stankovic et al., 2016; Zhao et al., 2018). Far less is known about how microglia interact with the vasculature in the healthy brain. With new evidence that microglia could be a conduit by which changes in peripheral immunity (e.g., microbiome, infection) affect CNS function (Hanamsagar and Bilbo, 2017; Hammond et al., 2018; Rothhammer et al., 2018; Zhao et al., 2018) and mounting evidence that an array of neurologic disorders have a vascular and microglial component (Daneman, 2012; Hammond et al., 2018; Zhao et al., 2018; Abdel-Haq et al., 2019; Lebovitz et al., 2018), a greater understanding of microglia-vascular interactions is necessary.

The neurovascular unit (NVU) is composed of endothelial cells, pericytes, vascular smooth muscle cells, astrocytes, macrophages, and neurons that connect the brain parenchyma to the cerebral vasculature. The interactions among these NVU cell types are important for a variety of physiological processes such as angiogenesis, vessel maintenance and permeability, metabolic support, and regulation of blood flow (McConnell et al., 2017; Brown et al., 2019). The development of the NVU begins around embryonic day 9.5 (E9.5) in mice, when specialized endothelial cells branch from vessels of the perineural vascular plexus to form capillaries that invade nearby neural tissue (Saili et al., 2017). Pericytes associate with endothelial cells as nascent vessels generate at E9.5 (Bauer et al., 1993; Armulik et al., 2010; Daneman et al., 2010; Yamanishi et al., 2012), and these interactions are critical to forming the BBB (Zlokovic, 2008; Daneman et al., 2010). Astrocytes are also a key component of the mature NVU. After the vasculature initially forms, astrocytes extend their processes to form endfeet over the course of postnatal development in rodents (Daneman et al., 2010). These astrocyte endfeet ultimately surround and ensheath the majority of the vasculature by adulthood, where they play roles in a variety of functions such as maintaining the BBB, providing metabolic support to neurons, and regulating blood flow (Abbott et al., 2006; Kimelberg and Nedergaard, 2010; Macvicar and Newman, 2015).

The vast majority of studies assessing interactions between microglia and the vasculature are in the context of disease. For example, microglia rapidly surround and contact the vasculature following breakdown of the BBB in the inflamed CNS (Stankovic et al., 2016; Zhao et al., 2018). One mechanism regulating these microglia-vascular interactions is the blood component fibrinogen and CD11b on microglia (Adams et al., 2007; Davalos et al., 2012). Reactive microglia can also influence the opening of the BBB by phagocytosing astrocyte endfeet or upregulating molecules such as VEGF, inducible nitric oxide synthase, and ROS (Stankovic et al., 2016; Zhao et al., 2018; Haruwaka et al., 2019). In the healthy brain, much less is known. Studies in rodents and humans have shown that microglia associate with the vasculature in the developing CNS, and live imaging in postnatal brain slices following traumatic injury or in embryonic mouse brain slices has suggested that microglia can migrate along the vasculature (Grossmann et al., 2002; Checchin et al., 2006; Monier et al., 2007; Fantin et al., 2010; Smolders et al., 2017). Microglia have also been suggested to regulate vascular growth and complexity in the developing hindbrain and retina (Checchin et al., 2006; Kubota et al., 2009; Fantin et al., 2010; Rymo et al., 2011; Dudiki et al., 2020). Together, these studies provide evidence that there is microglia-vascular cross talk, which requires further investigation in development, adulthood, and disease.

In the current study, we investigated microglia-vascular interactions in the healthy, developing, and adult cerebral cortex. Using confocal, super-resolution, expansion, and electron microscopy, we assessed the developmental regulation of associations between microglia and the vasculature and used fractalkine receptor (CX3CR1)-deficient mice to determine a role for this signaling in the timing of these interactions. Using in situ confocal and in vivo two-photon live imaging, we further assessed the dynamics of juxtavascular microglia in real time. Our data support a mechanism by which microglia migrate along the vasculature to colonize the developing brain, and the timing of these interactions is regulated by CX3CR1. This migratory behavior becomes restricted as astrocyte endfeet mature and suggests the establishment of a long-term niche for juxtavascular microglia in the adult brain.

\section{Materials and Methods}

Animals. Male and female mice were used for all experiments. $C \times 3 \mathrm{cr} \mathrm{I}^{-1-}$ mice $\left(C x 3 c r 1^{E G F P / E G F P}\right.$; stock \#005582) and C57BL6/J (stock \#000664) mice were obtained from The Jackson Laboratory. Heterozygous breeder pairs were set up for all experiments and wild-type (WT) and heterozygote littermates were used as controls with equal representation of males and females for each genotype. All experiments were performed in accordance with animal care and use committees and under National Institutes of Health guidelines for proper animal welfare.

Human prenatal brain collection and immunofluorescence microscopy. Deidentified prenatal human brain tissues were collected via the Department of Pathology Autopsy Service at the University of California, San Francisco, under the approval of the Committee on Human Research (Study \#12-08643). Brain tissues from four prenatal cases at 15, 18, 21, and 28 gestational weeks (GWs) were evaluated using standard neuropathologic examinations to rule out any gross or microscopic abnormalities. These autopsy cases, which all had postmortem intervals of $<48 \mathrm{~h}$, were fixed in freshly prepared $4 \%$ paraformaldehyde (PFA) and sampled at the level of the mammillary body. Following fixation in $4 \%$ PFA for $48 \mathrm{~h}$, brain samples were incubated with $20 \%$ sucrose solution and were frozen in embedding medium OCT for cryosectioning at $20 \mu \mathrm{m}$. For consistency, three to six consecutive sections were prepared from each sample and immunostained with anti-Ibal antibody (1:3000; Wako) and anti-CD31 antibody (1:200; R\&D Systems). Images of the ventricular and subventricular zones at the level of the frontal cortex were acquired on a Leica SP8 confocal microscope using a $40 \times$ [1.3 numerical aperture (NA)] objective lens.

Preparation of tissue for immunofluorescence microscopy. Mice were perfused with $1 \times$ HBSS-magnesium and HBSS-calcium (Thermo Fisher Scientific) before brain removal at indicated ages. For analysis of frontal and somatosensory cortex, brains were postfixed in $4 \%$ paraformaldehyde in $0.1 \mathrm{M}$ phosphate buffer (PB) for $4 \mathrm{~h}$. Brains were placed in $30 \%$ sucrose in $0.1 \mathrm{M} \mathrm{PB}$ and allowed to sink before sectioning. Sections were blocked in $10 \%$ goat serum, $0.01 \%$ Triton X-100 (TX-100) in $0.1 \mathrm{M} \mathrm{PB}$ for $1 \mathrm{~h}$ before primary immunostaining antibodies were applied overnight. Secondary antibodies were applied for $2 \mathrm{~h}$ the following day. All steps were conducted at room temperature with agitation. For structured illumination microscopy (SIM), sections were blocked in 3\% bovine serum albumin (BSA) and $0.01 \%$ Triton X-100 in $0.1 \mathrm{M} \mathrm{PB}$ for $1 \mathrm{~h}$ before primary immunostaining antibodies were applied for $48 \mathrm{~h}$ at $4^{\circ} \mathrm{C}$. Secondary antibodies were applied for $4 \mathrm{~h}$ at room temperature with agitation. The following antibodies were used: anti-P2RY12 (1:200; 
Butovsky Laboratory, Brigham and Women's Hospital, Harvard University), anti-PECAM (platelet endothelial cell adhesion molecule; 1:100; BioLegend), anti-aquaporin 4 (AQP4; 1:200; Millipore Sigma), anti-PDGFR $\beta$ (platelet-derived growth factor receptor $\beta$; 1:200; Thermo Fisher Scientific), anti-Lyve1 (1:200; Abcam), anti-smooth muscle actin (SMA; 1:200; Millipore Sigma), and anti-VGluT2 (vesicular glutamate transporter 2; 1:2000; Millipore Sigma).

Confocal microscopy. Immunostained sections were imaged on a Zeiss Observer Spinning Disk Confocal Microscope equipped with diode lasers $(405,488,594$, and $647 \mathrm{~nm})$ and Zen acquisition software (Zeiss). For microglia-vascular interaction, microglial density, nearest neighbor analysis, microglia association with $\mathrm{SMA}^{+}$or $\mathrm{SMA}^{-}$vessels and vascular density analyses, $20 \times$ single-optical plane tiled images of the frontal or somatosensory cortex were acquired for each animal. To create a field of view (FOV), each tiled image was stitched using Zen acquisition software. Two FOVs (i.e., tiled images) were acquired per animal. To note, anti-P2RY12 immunostaining was used to label microglia in wild-type animals, which was more difficult to visualize at lower magnification at older ages compared with EGFP-labeled microglia. As a result, for antiP2RY12 immunostained sections from P7-P28 animals, $1240 \times$ fields of view were acquired per animal with $76 z$-stack steps at $0.22 \mu \mathrm{m}$ spacing. For analysis of juxtavascular microglia that occur by chance, vascular diameter, juxtavascular association with branched/unsegmented vessels, primary processes aligned with vessels, astrocyte endfeet/juxtavascular microglia coverage on the vasculature, and vascular-associated microglia contacts with astrocytes, 6-12 40× fields of view were acquired from the frontal cortex per animal with $76 z$-stacks at $0.22 \mu \mathrm{m}$ spacing.

Juxtavascular microglia and microglia density analyses in the frontal and somatosensory cortices. Using the DAPI channel as a guide, a region of interest (ROI) was chosen in each cortical layer, I-VI, from each $20 \times$ stitched tiled image (10 ROIs per animal). Subsequent images were analyzed in Image (NIH). For anti-P2RY12, sections were acquired at $40 \times$, and a maximum intensity projection was made from each $z$-stack and was considered an ROI (12 per animal). The ROI areas were recorded. The same ROI was transposed on the microglial channel, and the cell counter ImageJ plugin was used to count the number of microglia in the ROI. The total density of microglia was then calculated by dividing the microglia number by the ROI area. To assess microglial association with the vasculature, the microglia and blood vessel channels were merged and the cell counter plugin was used to manually count the number of microglia with cell bodies directly apposed to blood vessels. Juxtavascular microglia were defined as microglia with at least $30 \%$ of their soma perimeter associated with blood vessels and soma centers that were within $10 \mu \mathrm{m}$ of the vessel. The percentage of juxtavascular microglia was calculated by summing the total number of microglia associated with vasculature divided by the total number of microglia within the ROI. To quantify the percentage juxtavascular microglia that occur by chance $6-1240 \times$ fields of view were analyzed. The number of associations between microglia and blood vessels was quantified, then the orientation of the blood vessel channel was horizontally flipped $180^{\circ}$ and the number of associations between microglia and vasculature was requantified. For each animal, data from the ROIs were averaged together to get a single average per animal for statistical analyses.

Juxtavascular microglia analysis within the barrel cortex. Juxtavascular microglia analysis in the barrel cortex was performed blinded to genotype. Images were analyzed in Image (NIH). From each tiled image from each animal, 12-18 images containing VGluT2 ${ }^{+}$barrels were cropped for subsequent analyses. From each cropped image, the individual channels were separated and, using the free-hand selection tool, each individual barrel was outlined. This ROI outlining the barrel was transposed to the microglia channel where the cell counter plugin was used to count the number of microglia in the barrels. The microglia and blood vessel channels were then merged, and the same ROI was transposed onto the merged image. The cell counter plugin was used to count the number of microglia in barrels associated with vasculature. Each individual barrel ROI was then cleared, leaving behind only the septa fluorescence, and the cell counter plugin was again used to count the number of microglia and the number of juxtavascular microglia in the septa. To calculate the percentage of juxtavascular microglia in the barrel cortex, the total numbers of juxtavascular microglia in the barrels and septa were summed and divided by the total number of microglia in the barrel and septa, respectively, for each ROI. The total microglia in barrels and septa, regardless of vascular association, were also calculated. All numbers across 12-18 cropped images were then averaged for a given animal before statistical analyses.

Vascular density analysis. Density analysis was performed blinded to genotype from the same tiled and stitched $20 \times$ images used for microglia-vascular association analyses. Using Image (NIH) software, the blood vessel channel was thresholded manually and the total blood vessel area was measured. Vascular density was calculated by dividing the blood vessel area by the area of the ROI. For each animal, the vascular density was averaged across all ROIs in the two FOVs to get a single average per animal for statistical analyses.

Nearest neighbor analysis. Stitched $20 \times$ images, used for microgliavascular association analyses, were used to determine nearest neighbor. Using Image (NIH) software, an ROI was drawn around the cortex. The microglia channel was thresholded so only somas were selected, the number of microglia was calculated using the analyze particles function, and microglia were manually annotated as juxtavascular or vascularunassociated microglia. The distance between nearest neighbor was calculated using the nearest neighbor distances plugin. For each animal, the nearest neighbor distance for juxtavascular and vascular-unassociated microglia was averaged across the two FOV to get a single average per animal for statistical analysis.

Microglial association with $\mathrm{SMA}^{+}$or $S M A^{-}$vessels analysis. Using the DAPI channel as a guide, an ROI was chosen in each cortical layer, I-VI, from each $20 \times$ stitched tiled image (10 ROIs per animal). Subsequent images were analyzed in ImageJ (NIH). The same ROI was transposed on the microglial, PDGFR $\beta$, and SMA channel, and the cell counter ImageJ plugin was used to count the total number of microglia, the number of juxtavascular microglia, and the number of juxtavascular microglia associated with $\mathrm{SMA}^{+}$or $\mathrm{SMA}^{-}$vessels in the ROI. The percentage of juxtavascular microglia associated with $\mathrm{SMA}^{+}$or $\mathrm{SMA}^{-}$vessels was quantified by dividing the number of microglia on $\mathrm{SMA}^{+}$or $\mathrm{SMA}^{-}$vessels by the total number of juxtavascular microglia. For each animal, data from the ROIs were averaged together to get a single average per animal for statistical analyses.

Vascular diameter analysis. Using Imaris software (Bitplane), the diameter of the vessel was measured in 3D at microglial soma association points from $40 \times$ images ( 12 per animal). For each animal, data from the 12 images were averaged together to get a single average per animal for statistical analysis.

Primary process and branched/unsegmented vessel analyses. Using Image (NIH), the total number of primary processes, the number of primary processes aligned parallel with vessels, and whether the juxtavascular microglia were associated with a vessel branch point was calculated from $40 \times$ images ( 6 per animal, $n=3-4$ animals). The percentage of primary processes aligned with vessels was calculated by dividing the number of primary processes aligned parallel and associated with vessels by the total number of primary processes. The percentage of juxtavascular microglia associated with branched/unsegmented vessels was calculated by dividing the number of juxtavascular microglia associated with branched or unsegment vessels by the total number of juxtavascular microglia. For each animal, data from six images were averaged together to get a single average per animal for statistical analysis.

Acute slice time-lapse imaging. Mice were given a retro-orbital injection of Texas Red-labeled dextran (Thermo Fisher Scientific) $10 \mathrm{~min}$ before being killed to label vasculature. Mice were killed at $\mathrm{P} 7$ or greater than or equal to P120, brains were isolated and sectioned coronally at a thickness of $300 \mu \mathrm{m}$ using a Leica VT1200 vibratome in oxygenated $37^{\circ}$ $\mathrm{C}$ artificial CSF (ACSF). Slices were mounted on a MatTak glass bottom microwell dish and placed in a Zeiss Observer Spinning Disk Confocal Microscope equipped with diode lasers $(405,488,594$, and $647 \mathrm{~nm})$ and Zen acquisition software (Zeiss). Image acquisition started after a minimum of $30 \mathrm{~min}$ of tissue equilibration at $37^{\circ} \mathrm{C}$ with $5 \% \mathrm{CO}_{2}$ and within 2 $\mathrm{h}$ of decapitation. Oxygenated ACSF was continuously perfused over the slices at a rate of $1.5-2 \mu \mathrm{m} / \mathrm{min}$ for the duration of equilibration and imaging. Per animal, one field of view was imaged every $5 \mathrm{~min}$ over $6 \mathrm{~h}$ on an inverted Zeiss Observer Spinning Disk Confocal and a $20 \times$ 
objective. The $z$-stacks spanning 50-60 $\mu \mathrm{m}$, with serial optical sections of 1.5-2 $\mu \mathrm{m}$, were recorded from a minimal depth of $30 \mu \mathrm{m}$ beneath the surface of the slice to avoid cells activated by slicing.

In vivo two-photon time-lapse imaging. Cranial window surgeries were performed as previously described within the visual cortex $(2.5 \mu \mathrm{m}$ lateral and $2.0 \mu \mathrm{m}$ posterior from bregma; Goldey et al., 2014). One week after surgery, mice were head fixed to a custom-built running wheel and trained to run while head restrained for increasing time intervals several days a week. Two weeks postsurgery, long-term two-photon live imaging began. Mice were given a retro-orbital injection of Texas Red-labeled dextran (Thermo Fisher Scientific) 10 min before imaging and were head restrained on a custom-built running wheel, which was positioned directly under the microscope objective. Images were acquired with a $20 \times$ water-immersion objective (NA 1.0; Zeiss) on a Zeiss Laser Scanning 7 MP Microscope equipped with a tunable coherent Chameleon Ultra II multiphoton laser and BiG detector. Three different ROIs were taken at least $75 \mu \mathrm{m}$ below the surface of the brain, with $z$-stacks spanning $45-65 \mu \mathrm{m}$ with a step size of $2.5 \mu \mathrm{m}$ for each animal. On the first day of imaging, each ROI was imaged every 5 min over $2 \mathrm{~h}$. The same ROIs were then imaged once (single $z$-stack) on the following days after the first imaging session: $1,3,7,10,14,17,21,24,28$, 35 , and $42 \mathrm{~d}$. For each imaging day, the ROIs from day 0 of imaging were identified based on the vascular structure.

Migration tracking and analysis. Image processing and microglial soma motility/migration tracking were performed using Image (NIH). Time series were first corrected for 3D drift using the 3D drift correction plugin (Parslow et al., 2014), and migration was tracked using the TrackMate plugin (Tinevez et al., 2017). For each developmental time point, 10-12 juxtavascular and vascular-unassociated microglia were analyzed per animal ( $n=4$ mice per developmental time point). Only cells remaining in the field of view for $6 \mathrm{~h}$ were included in the analysis. The average soma motility (in micrometers per hour) was calculated by measuring the displaced distance of the microglial soma between time $=0 \mathrm{~min}$ and time $=360 \mathrm{~min}$ and dividing by the duration of the imaging session. Juxtavascular distance migrated was calculated by measuring the displaced distance of the microglial soma between time $=0 \mathrm{~min}$ and time $=360 \mathrm{~min}$. Juxtavascular migration trajectory was calculated by measuring the angle between the blood vessel and juxtavascular microglia soma along the longest, continuous stretch of motility on the vessel. The percentage of cells within each binned category (motility, distance traveled, and trajectory) was calculated by dividing the number microglia within each category by the total number of microglia. For each animal, data from each analyzed cell were averaged together to get a single average per animal for statistical analysis.

In vivo tracking of juxtavascular microglia motility and long-term juxtavascular microglia were performed using ImageJ (NIH). Time series were first corrected for $3 \mathrm{D}$ drift using the $3 \mathrm{D}$ drift correction plugin (Parslow et al., 2014), and migration was tracked using the TrackMate plugin (Tinevez et al., 2017). To calculate the percentage of microglia stationary over $2 \mathrm{~h}$, the number of stationary juxtavascular and vascularunassociated microglia was divided by the total number of microglia. To calculate the percentage of original juxtavascular microglia that remain on vessels over $42 \mathrm{~d}$, the number of juxtavascular microglia on day 0 was calculated. For each subsequent day, the number of these original juxtavascular microglia that were still associated with the vasculature was determined and divided by the number of original juxtavascular microglia on day 0 . For each animal, data were analyzed from three ROIs and averaged together to get a single average per animal for statistical analysis.

Astrocyte endfeet and juxtavascular microglia coverage analysis. Using Imaris software (Bitplane), the astrocyte endfeet, microglia, and vessel channels were 3D rendered from $40 \times$ images ( 6 per animal). The astrocyte and microglia channels were then masked onto the vessel channel, and the masked astrocyte channel and microglia were 3D rendered. Volumes of the 3D-rendered vessel channel, masked astrocyte endfeet channel, and masked microglia channel were recorded. The percentage of blood vessels covered by astrocyte endfeet was calculated by dividing the blood vessel volume by the masked astrocyte endfeet volume. The percentage of blood vessels covered by juxtavascular microglia was calculated by dividing the blood vessel volume by the masked microglia volume.
Uncovered vessel volume was calculated by adding the masked astrocyte endfeet and juxtavascular microglia volume and subtracting from the vessel volume. The percentage of juxtavascular microglia associated with vessels was calculated by dividing the microglial volume by the masked microglial volume. For each animal, data from the six images were averaged together to get a single average per animal for statistical analysis.

Juxtavascular microglia-astrocyte association. Analysis was performed using the same images used for astrocyte endfeet coverage analysis in Imaris (Bitplane). The microglia were 3D rendered, masked onto the blood vessel and astrocyte endfeet channel, and the volume of the masked microglial channel was recorded. The percentage of juxtavascular microglia associated with blood vessels only, vessels and astrocyte endfeet, or astrocyte endfeet only was calculated by summing the number of microglia associated with vessels only, vessels and astrocyte endfeet, or astrocyte endfeet only and dividing by the total number of juxtavascular microglia. For each animal, data from the six images were averaged together to get a single average per animal for statistical analysis.

Expansion microscopy. Expansion microscopy (ExM) was performed as previously described (Asano et al., 2018) with slight modification. Briefly, $80 \mu \mathrm{m}$ floating sections were blocked in $0.5 \%$ BSA and $0.3 \%$ TX-100 for $1 \mathrm{~h}$ at room temperature. Primary antibodies, anti-aquaporin 4 (1:200; Millipore Sigma), anti-PDGFR $\beta$ (1:100; Thermo Fisher Scientific), and anti-GFP (1:200; Abcam) were incubated in $0.5 \% \mathrm{BSA}$ and $0.3 \% \mathrm{TX}-100$ at $4^{\circ} \mathrm{C}$ for 4 nights. Secondary antibodies were added at 1:200 dilutions overnight at room temperature. The expansion microscopy protocol (Basic Protocol 2) was then followed as published in the study by Asano et al. (2018).

Structured illumination microscopy. SIM was performed using a GE DeltaVision OMX V4 Microscope with pCO.edge sCMOS (scientific complementary metal-oxide-semiconductor) cameras and an Olympus $60 \times 1.42$ NA objective. Samples were mounted in Prolong Glass mounting media with \#1.5 coverslips and imaged using 1.516 refractive index immersion oil. Image processing was completed using the GE softWorx software, and image quality was determined using the SIMcheck plugin in ImageJ. SIM figures were produced in ImageJ (NIH).

Scanning electron microscopy. Mice were perfusion fixed in $2.5 \%$ glutaraldehyde and $2 \%$ paraformaldehyde in $0.1 \mathrm{~m}$ sodium cacodylate buffer at $\mathrm{pH} 7.4$ (Science Services). Brains were dissected, vibratome sectioned, and immersion fixed for $24 \mathrm{~h}$ at $4^{\circ} \mathrm{C}$. We applied a rOTO (reduced osmium-thiocarbohydrazide-somium) staining procedure adapted from Tapia et al. (2012). Briefly, the tissue was washed and postfixed in $2 \%$ osmium tetroxide (EMS) and $2 \%$ potassium hexacyanoferrate (SigmaAldrich) in $0.1 \mathrm{~m}$ sodium cacodylate buffer. After washes in buffer and water, the staining was enhanced by reaction with $1 \%$ thiocarbohydrazide (Sigma-Aldrich) for $45 \mathrm{~min}$ at $50^{\circ} \mathrm{C}$. The tissue was washed in water and incubated in $2 \%$ aqueous osmium tetroxide. All osmium incubation steps were conducted over $90 \mathrm{~min}$ with substitution by fresh reagents after $45 \mathrm{~min}$, respectively. To further intensify the staining, $2 \%$ aqueous uranyl acetate was applied overnight at $4^{\circ} \mathrm{C}$ and subsequently warmed to $50^{\circ} \mathrm{C}$ for $2 \mathrm{~h}$. The samples were dehydrated in an ascending ethanol series and infiltrated with LX112 (LADD). The samples were flat embedded into gelatin capsules (Science Services) and cured for $48 \mathrm{~h}$. The block was trimmed by $200 \mu \mathrm{m}$ at a $90^{\circ}$ angle on each side using a TRIM90 diamond knife (Diatome) on an ATUMtome (RMC Powertome). Consecutive sections were taken using a $35^{\circ}$ ultra-diamond knife (Diatome) at a nominal cutting thickness of $100 \mathrm{~nm}$ and collected on freshly plasma-treated (custom-built, based on Pelco easiGlow, adopted from Mark Terasaki, University of Connecticut Health Center, Farmington, CT) CNT tape (Kubota et al., 2018). We collected 450 (P5) and 550 (P56) cortical sections, covering a thickness of $45-55 \mu \mathrm{m}$ in depth. Tape strips were mounted with adhesive carbon tape (Science Services) onto 4 inch silicon wafers (SIEGERT WAFER) and grounded by additional adhesive carbon tape strips (Science Services). Electron microscopy (EM) micrographs were acquired on a Crossbeam Gemini 340 SEM (Zeiss) with a four-quadrant backscatter detector at $8 \mathrm{kV}$. In ATLAS5 Array Tomography (Fibics), the whole wafer area was scanned at $3000 \mathrm{~nm} /$ pixel to generate an overview map. The entire ultrathin section areas of one wafer [314 sections (P5), 279 sections (P56)] were scanned at $100 \times 100 \times$ 


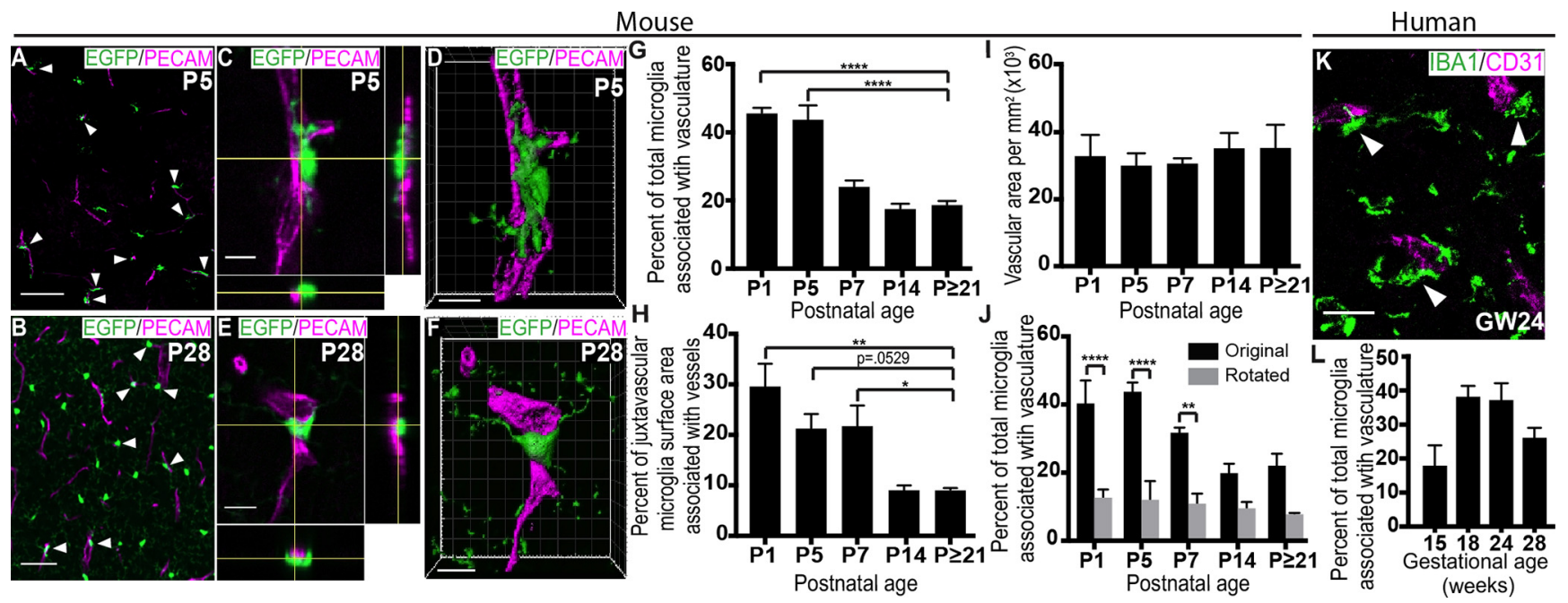

Figure 1. A high percentage of microglia is juxtavascular during early postnatal development. $A, B$, Representative low-magnification tiled images of microglia (green, EGFP) associated with vasculature (magenta, anti-PECAM) in the P5 ( $\boldsymbol{A})$ and P28 $(\boldsymbol{B})$ frontal cortex. Filled arrowheads denote juxtavascular microglia. Scale bars: $\boldsymbol{A}, 100 \mu \mathrm{m} ; \boldsymbol{B}, 50 \mu \mathrm{m}$. $\boldsymbol{C}, \boldsymbol{D}$, High-magnification, orthogonal view $(\boldsymbol{C})$ and 3D reconstruction and surface rendering $(\boldsymbol{D})$ of juxtavascular microglia in the P5 frontal cortex (Movie 1). Scale bars, $10 \mu \mathrm{m}$. $\boldsymbol{E}, \boldsymbol{F}$, Orthogonal $(\boldsymbol{E})$ and 3D reconstruction and surface rendering $(\boldsymbol{F})$ of a juxtavascular microglia in the P28 frontal cortex (Movie 2). Scale bars, $10 \mu \mathrm{m}$. $\mathbf{G}$, The percentage of the total microglia population associated with vasculature over development in the frontal cortex. One-way ANOVA with Dunnett's post hoc test; comparison with P21 or greater, $n=4$ littermates per developmental time point, ${ }^{* * * *} p<0.0001$. $\boldsymbol{H}$, Quantification of the percentage of juxtavascular microglia surface area associating with vessels over development in the frontal cortex in 3D reconstructed confocal images. One-way ANOVA with Dunnett's post hoc test; comparison with P21 or greater, $n=3$ littermates per developmental time point, ${ }^{*} p=0.0445,{ }^{* *} p=.0025$. $I$, Vascular density over development in the frontal cortex. One-way ANOVA with Dunnett's post hoc test; comparison with P21 or greater, $n=4$ littermates per developmental time point. $J$, The percentage of total microglia associated with vasculature that occurs by chance in the frontal cortex over development. Two-way ANOVA with a Sidak's post hoc test; $n=4$ littermates per developmental time point, ${ }^{* *} p=0.0022$, ${ }^{* * *} p<.0001$. $\boldsymbol{K}$, Representative image of microglia (green, anti-IBA1) associated with vasculature (magenta, anti-CD31) in GW 24 in the ventricular zone (VZ) and subventricular zone (SVZ) at the level of the human frontal cortex. Filled arrowheads denote juxtavascular microglia. Scale bar, $20 \mu \mathrm{m}$. $L$, Quantification of the percentage of total microglia associated with vasculature in the human brain. One-way ANOVA across all ages, $p=0.0544, n=3-6$ fields of view per gestational age (1 individual specimen per gestational age). All error bars represent \pm SEM.

$10^{0} \mathrm{~nm}^{3}\left[465 \times 638 \mu \mathrm{m}^{2}\right.$ (P5), $1249 \times 707 \mu \mathrm{m}^{2}$ (P56)]. After alignment in Fiji TrakEM2 (Cardona et al., 2012) areas that contained microglia in close proximity to blood vessels [148 $\times 136 \times 16 \mu \mathrm{m}^{3}$ (P5), $193 \times 186 \times 12 \mu \mathrm{m}^{3}$ (P56)] were selected for high-resolution acquisition. We collected 29 total $2 \mathrm{D}$ micrographs $\left(10 \times 10 \mathrm{~nm}^{2}\right)$ from $n=3$ animals at P5 and 11 total micrographs from $n=3$ animals at P56. From each age, one juxtavascular microglia was identified and selected to generate a $3 \mathrm{D}$ volume $\left(10 \times 10 \times 10^{0} \mathrm{~nm}^{3}\right)$. The image series were aligned in TrakEM2 using a series of automated and manual processing steps. For the P5 and P56 image series, segmentation and rendering were performed in VAST (Volume And Segmentation Tool; Berger et al., 2018). We used Blender to render the two 3D models (Blender Foundation, 2018). The percentage of juxtavascular microglia contacting basal lamina was calculated using ImageJ (NIH). Single optical plane images were opened, the perimeter area of microglia was measured, and the length of contact between microglia and the basal lamina was measured. The percentage of juxtavascular microglia contacting the basal lamina was calculated by dividing the perimeter of the microglia by the length of contact between microglia and basal lamina.

Experimental design and statistical analyses. GraphPad Prism 7 (GraphPad Software) provided the platform for all statistical and graphical analyses. The ESD (extreme studentized deviate) method was run for each ROI per animal to identify outliers. Significant outliers were removed before analyses. Analyses included Students $t$ test when comparing two conditions or one-way ANOVA followed by Dunnett's post hoc analysis or two-way ANOVA followed by Sidak's or Tukey's post hoc analyses (indicated in figure legends in Results).

\section{Results}

A high percentage of microglia is juxtavascular during development

During rodent and human embryonic development, microglia somas have been described to be in close association with blood vessels (i.e., juxtavascular microglia; Checchin et al., 2006; Monier et al., 2007; Fantin et al., 2010). We assessed microglial association with the vasculature over an extended developmental time course across postnatal development. Microglia were labeled using transgenic mice that express EGFP under the control of the fractalkine receptor CX3CR1 ( $\left.C \times 3 \mathrm{cr} 1^{E G F P /+}\right)$. The vasculature was labeled with an antibody against PECAM. To start, we focused our analyses in the frontal cortex. Juxtavascular microglia were defined as microglia with at least $30 \%$ of their soma perimeter in association with blood vessels and soma centers that were within $10 \mu \mathrm{m}$ of the vessel, which we confirmed with orthogonal views and $3 \mathrm{D}$ surface rendering (Fig. $1 A-F$, Movies 1 , 2). Juxtavascular microglia were further distinguished from perivascular macrophages by their morphology with processes emanating from their soma and higher levels of EGFP. Using these criteria, we found the percentage of total microglia associated with vasculature and microglial soma surface area associated with the vessel was higher during early development than in older animals in the frontal cortex (Fig. $1 G, H$; one-way ANOVA with Dunnett's post hoc; comparison with P21 or greater, $n=3-4$ littermates per developmental time point, ${ }^{\star} p=0.0445,{ }^{* *} p=0.0025$, $\left.{ }^{* * * *} p<0.0001\right)$. We further found that a higher percentage of the total microglial population were juxtavascular at P1-P5 in the frontal cortex (Fig. 1G; one-way ANOVA with Dunnett's post hoc test; comparison with P21 or greater, $n=4$ littermates per developmental time point, $\left.{ }^{* * *} p<0.0001\right)$. These results were independent of sex (data not shown). The percentage of association dropped to $<20 \%$ by P14 and was maintained at later ages. We confirmed that this developmental regulation of juxtavascular microglia was independent of changes in vasculature density over development. While the total vascular content of the cortex increases as the brain grows, the density of the blood vessels within 


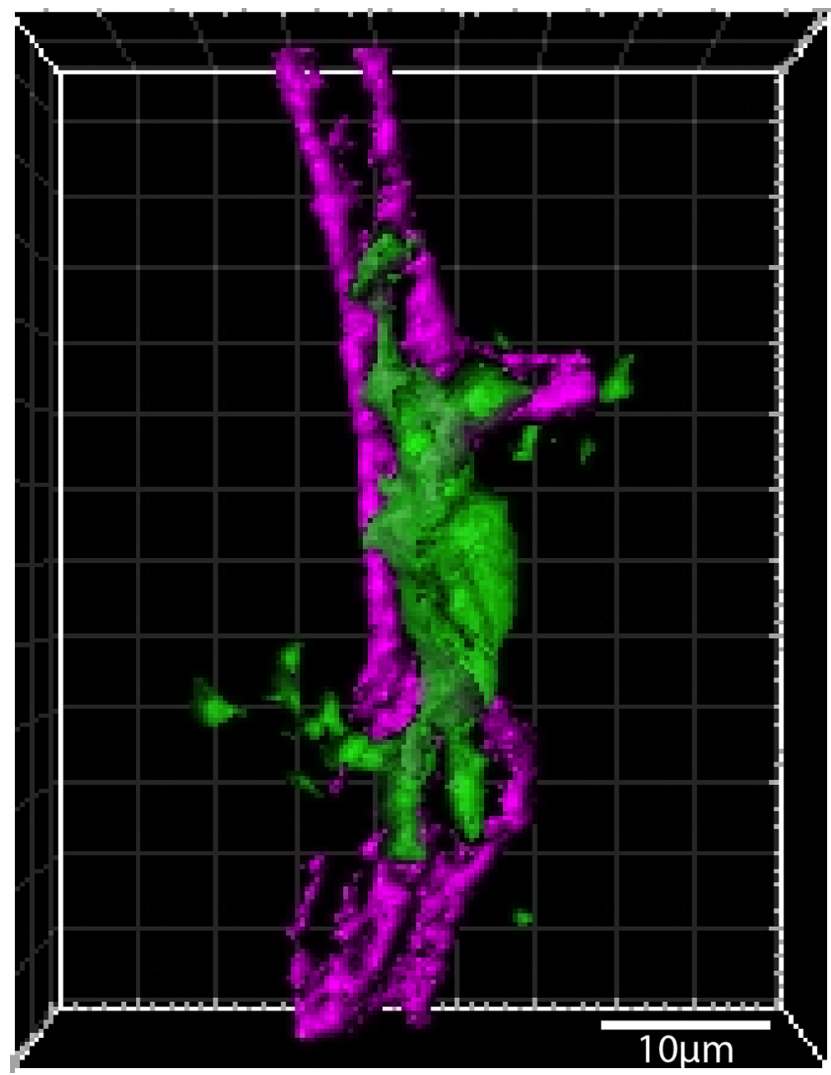

Movie 1. 3D rendering of juxtavascular microglia in the early postnatal frontal cortex. 3D reconstruction and surface rendering of juxtavascular microglia (green, EGFP) associated with blood vessels (magenta, anti-PECAM) in the P5 frontal cortex. Yellow denotes association area between microglia and blood vessels. [View online]

a given field of view is unchanged across development (Fig. 1I; one-way ANOVA with Dunnett's post hoc; comparison with P21 or greater, $n=4$ littermates per developmental time point). Moreover, developmental changes in juxtavascular microglia were likely not because of chance encounters with the vasculature, as rotating the blood vessel images $180^{\circ}$ resulted in reduced percentages of association with the vasculature over development (Fig. 1j; two-way ANOVA with a Sidak's post hoc test; $n=4$ littermates per developmental time point, ${ }^{* *} p=0.0022,{ }^{* * * *} p<0.0001$ ). Consistent with the results in mouse, the ventricular and subventricular zones of the prenatal human brain at the level of the frontal cortex also showed a high percentage of juxtavascular microglia. This association in the developing human brain peaked at $18-24$ GWs where $38 \%$ of total microglia were juxtavascular (Fig. $1 K, L$; one-way ANOVA across all ages, $p=0.0544, n=1$ specimen per gestational age) - a percentage similar to what we identified in early postnatal mice. Together, these data demonstrate that a large percentage of the total microglia are juxtavascular in the early postnatal mouse and prenatal human brain.

Juxtavascular microglia are largely associated with capillaries in the early postnatal cortex

While previous work has described similar high association of microglia with the vasculature in the embryonic/prenatal brain, these studies did not use markers to distinguish microglia from perivascular macrophages (Checchin et al., 2006; Monier et al., 2007; Fantin et al., 2010). Therefore, we next sought to confirm that vascular-associated $\mathrm{EGFP}^{+}$cells were, indeed, microglia, versus perivascular macrophages, and to determine the types of

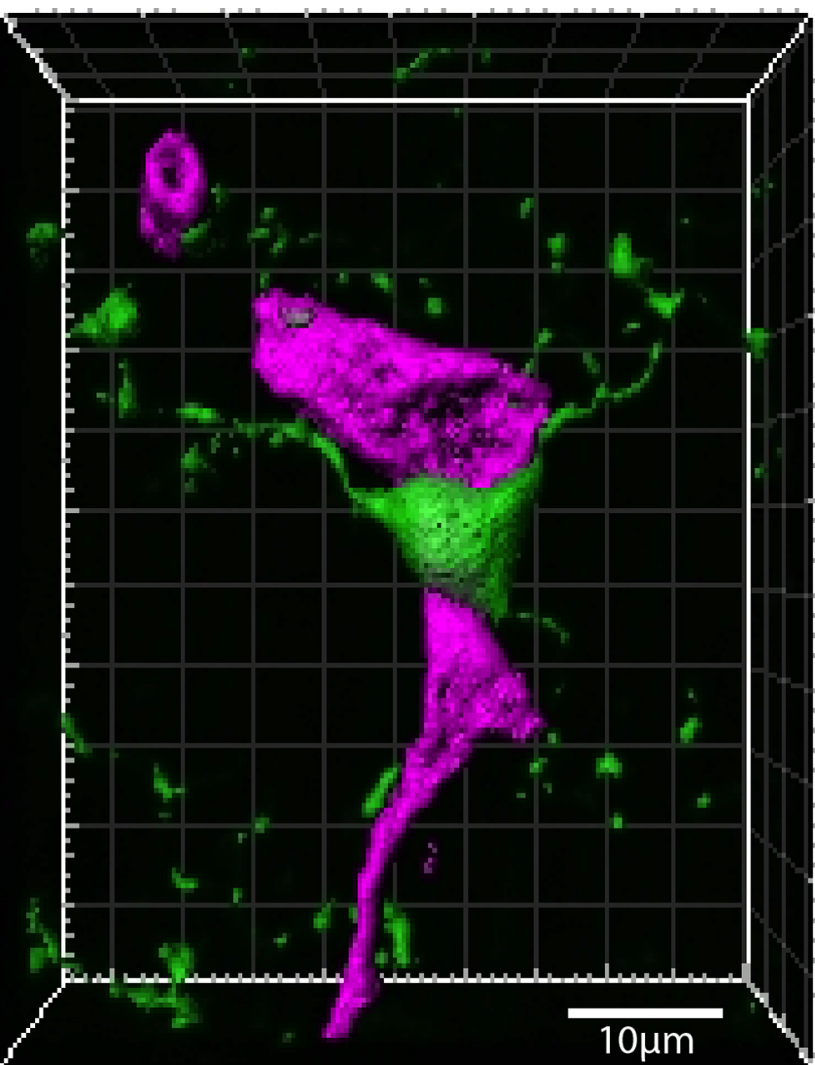

Movie 2. 3D rendering of juxtavascular microglia in the P28 frontal cortex. 3D reconstruction and surface rendering of juxtavascular microglia (green, EGFP) associated with blood vessels (magenta, anti-PECAM) in the P28 frontal cortex. Yellow denotes association area between microglia and blood vessels. [View online]

vessels associated with juxtavascular microglia. We found that the juxtavascular $\mathrm{EGFP}^{+}$cells that we initially identified as microglia based on their larger numbers of processes and higher levels of EGFP (Figs. 1, 2A,B, filled arrowheads) were also positive for the microglia-specific marker P2RY12 (Fig. 2A, filled arrowhead) and negative for the perivascular macrophage-specific marker LYVE1 (Fig. 2B, unfilled arrowheads; Butovsky et al., 2014; Zeisel et al., 2015). Using anti-P2RY12 to label microglia in wild-type mice, or EGFP in $C \times 3 c r 1^{E G F P /+}$ mice, which are heterozygotes for $C x 3 \mathrm{cr} 1$, we obtained similar percentages of juxtavascular microglial and vascular density (Fig. 2C,D; twoway ANOVA with a Sidak's post hoc test; $n=3-4$ littermates per genotype per developmental time point), confirming that results were independent of the microglial labeling technique. We also found that these juxtavascular microglia were associated largely along unsegmented vessels, rather than branch points, across postnatal development (Fig. 2E; two-way ANOVA with a Sidak's post hoc test; $n=3-4$ littermates per developmental time point, $\left.{ }^{\star} p=0.0118,{ }^{\star * *} p=0.0003,{ }^{* * * *} p<0.0001\right)$. We next assessed what types of vessels were associated with juxtavascular microglia, using a combination of parameters. Capillaries are $\leq 8 \mu \mathrm{m}$ in diameter and are $\mathrm{SMA}^{-}$and PDGFR $\beta^{+}$(Grant et al., 2019; Mastorakos and Mcgavern, 2019). Arterioles are $>8 \mu \mathrm{m}$ in diameter and are $\mathrm{SMA}^{+}$, and a subset of precapillary arterioles are also PDGFR $\beta^{+}$(Grant et al., 2019). Using these markers, we identified that juxtavascular microglia were largely associated with capillaries $\left(\leq 8 \mu \mathrm{m}, \mathrm{SMA}^{-}, \mathrm{PDGFR} \beta^{+}\right.$; Fig. $2 \mathrm{~F}-\mathrm{H}$; two-way ANOVA with a Sidak's post hoc test; $n=3$ littermates per genotype per developmental time point, $\left.{ }^{* * *} p<0.0001\right)$. These 

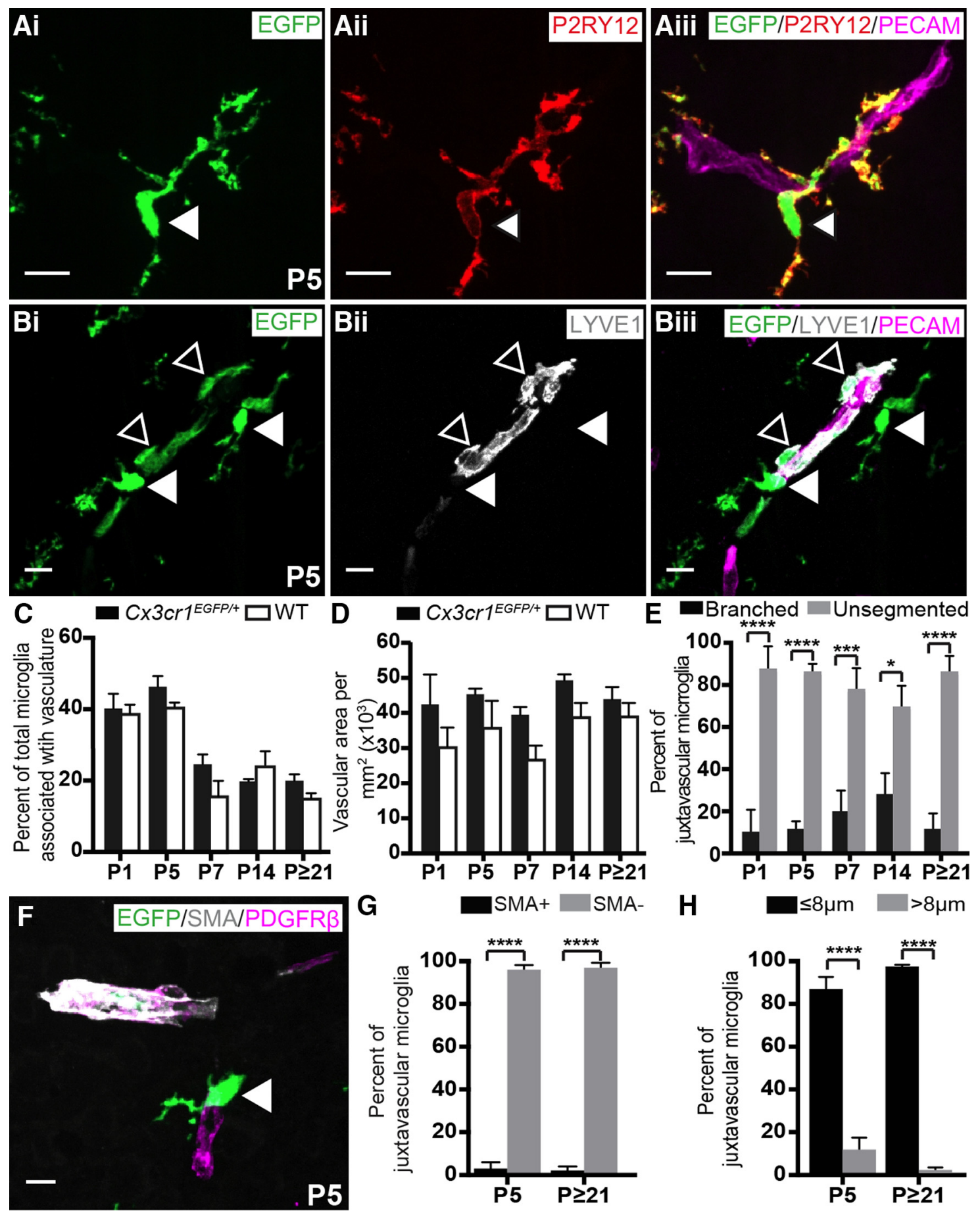

Figure 2. Juxtavascular microglia predominantly associate with capillaries in the postnatal cortex. Ai-Aiii, A representative image of a juxtavascular microglia (filled arrowhead) in the P5 frontal cortex. Microglia are labeled using the $\left(X 3 \mathrm{Cr}^{\mathrm{EGFP} /+}\right.$ reporter mouse (green; $\boldsymbol{A i}$ ) and immunolabeling for a microglia-specific marker anti-P2RY12 (red; Aii). The vasculature is labeled with anti-PECAM (magenta) in the merged image (Aiii). Scale bar, $10 \mu \mathrm{m}$. B, A representative image of LYVE1-negative microglia (green, EGFP; filled arrowheads; Bi) and LYVE1-positive perivascular macrophages (gray, anti-LYVE1; unfilled arrowheads; Bii) associated with vasculature (magenta, anti-PECAM; Biii) in the P5 frontal cortex. Scale bar, $10 \mu$ m. $C$, Quantification of juxtavascular microglia across development labeled either with EGFP in CX3cr1 ${ }^{\text {EGFP/+ }}$ mice (black bars) or anti-P2RY12 in wild-type (WT) mice (white bars) frontal cortices. Tw0-way ANOVA with a Sidak's post hoc test; $n=3-4$ littermates per genotype per developmental time point. D, Quantification of vascular density in (X3cr $1{ }^{E G F P /+}$ (black bars) and WT (white bars) frontal cortices over development. Two-way ANOVA with a Sidak's post hoc test; $n=3-4$ littermates per genotype per developmental time point. $\boldsymbol{E}$, Quantification of the percentage of juxtavascular microglia associated with branched (black bars) or unsegmented (gray bars) vessels. Two-way ANOVA with a Sidak's post hoc test; $n=3-4$ littermates per developmental time point, ${ }^{*} p=0.0118$, ${ }^{* * *} p=0.0003,{ }^{* * * *} p<0.0001$. $\boldsymbol{F}$, A representative image of a juxtavascular microglia (green, EGFP; filled arrowhead) associated with smooth muscle cell actin-negative (gray, SMA) capillaries (magenta, PDGFR $\beta$ ) in the P5 frontal cortex. Scale bar, $10 \mu \mathrm{m}$. G, Quantification of the percentage of juxtavascular microglia associated with SMA ${ }^{+}$or SMA ${ }^{-}$vessels at P5 and P21 or greater in the frontal cortex. Two-way ANOVA with a Sidak's post hoc test; $n=3$ littermates per genotype per developmental time point, ${ }^{* * * *} p<0.0001$. $\boldsymbol{H}$, Quantification of the percentage of juxtavascular microglia associated with vessels $\leq 8$ and $>8 \mu \mathrm{m}$ at P5 and P21 or greater in the frontal cortex. Two-way ANOVA with a Sidak's post hoc test; $n=4$ littermates per genotype per developmental time point, ${ }^{* * * *} p<0.0001$. All error bars represent \pm SEM. 


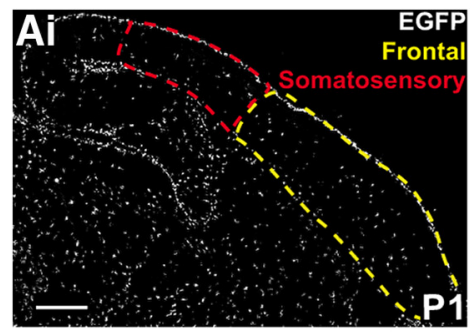

B
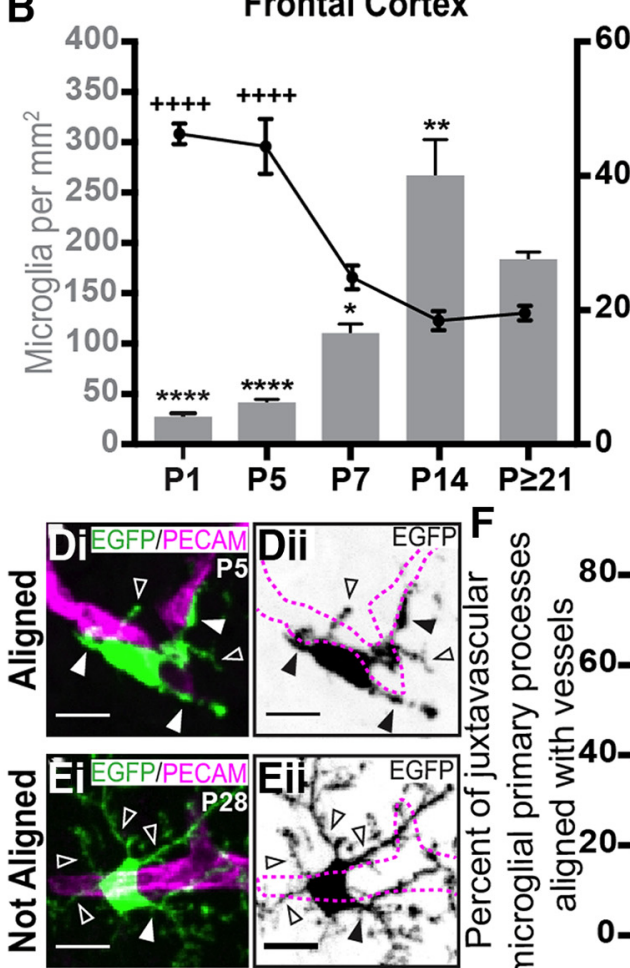

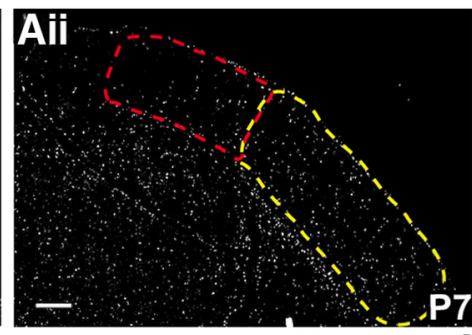

C

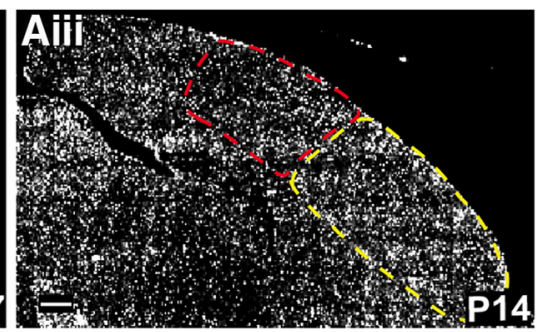

Somatosensory Cortex
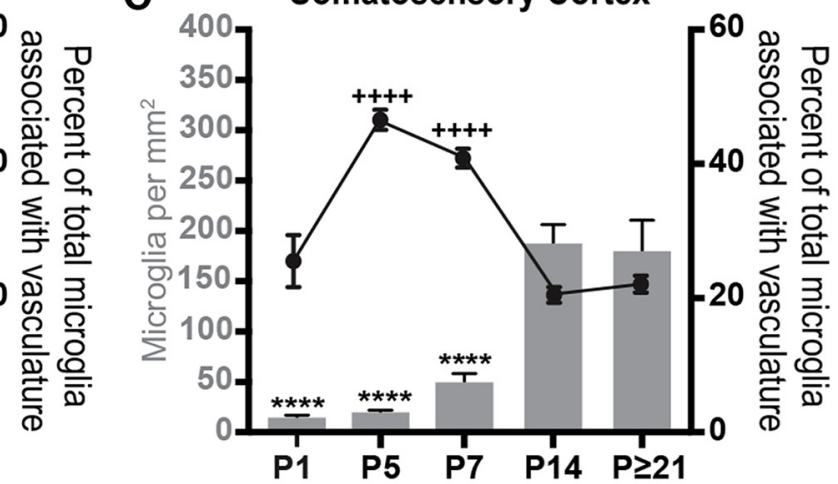

Frontal Cortex

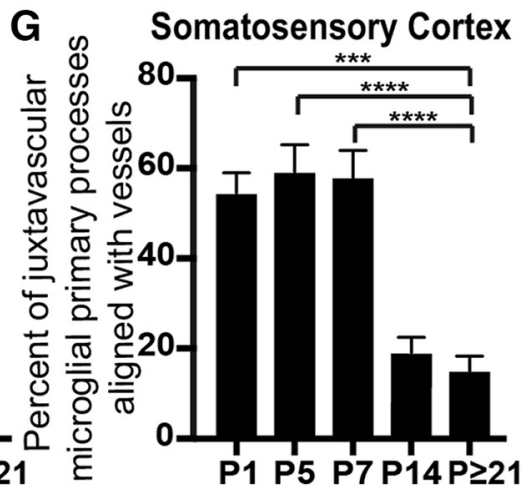

Figure 3. Microglia associate and align with vasculature as they colonize the cortex in a rostral-to-caudal gradient. Ai-Aiii, Tiled sagittal sections of a P1 (Ai), P7 (Aii), and P14 (Aiii) $C \times 3 C r 7^{E G F P /+}$ brain. The dotted yellow and red lines outline the frontal and somatosensory cortex, respectively. Scale bars, $400 \mu \mathrm{m}$. $\boldsymbol{B}, \boldsymbol{C}$, Left, $y$-Axis and gray bars: quantification of microglial density over development in the frontal cortex $(\boldsymbol{B})$ and somatosensory cortex (C). One-way ANOVA with Dunnett's post hoc test; comparison with P21 or greater, $n=4$ littermates per developmental time point, ${ }^{*} p=0.0182,{ }^{* *} p=0.0062,{ }^{* * *} p<0.0001$. Right, $y$-Axis and black line graphs: the percentage of the total microglia population associated with vasculature over development in the frontal cortex $(\boldsymbol{B})$ and somatosensory cortex $(\boldsymbol{C})$. Note, data corresponding to the percentage of juxtavascular microglia in the frontal cortex $(\boldsymbol{C}$, line graph) are the same as presented in Figure 1G. One-way ANOVA with Dunnett's post hoc test; comparison with P21 or greater, $n=4$ littermates per developmental time point, $++++p<0.0001$. Di-Eii, Representative images of juxtavascular microglia (EGFP; Di, Ei, green, Dii, Eii, black) primary processes aligned parallel with vessels (Di, Dii, magenta, anti-PECAM) in the P5 frontal cortex, which were largely not aligned at P28 (Ei, Eii). Filled arrowheads denote processes aligned parallel to the vessel and unfilled arrowheads denote those microglial processes that are not aligned with the vessel. The dotted magenta line in Dii and Eii outline the vessel in Di and $\boldsymbol{E}$. Scale bars, $10 \mu \mathrm{m}$. F, G, Quantification of the percentage of juxtavascular primary processes that are aligned parallel with vessels in the frontal $(\boldsymbol{F})$ and somatosensory $(\boldsymbol{G})$ cortices over development. One-way ANOVA with Dunnett's post hoc test; comparison with P21 or greater, $n=4$ littermates per developmental time point; frontal cortex: ${ }^{* *} p=0.0021(\mathrm{P} 1),{ }^{* *} p=0.0033$ (P5), somatosensory cortex: $\left.{ }^{* *} p=0.0003,{ }^{* * *} p<0.0001\right)$. All error bars represent \pm SEM.

experiments establish that a large percentage of bona fide microglia is associated with unsegmented capillaries in the postnatal cerebral cortex, and these percentages are similar in wild-type and $C x 3 c r 1^{E G F P /+}$ mice.

High percentages of juxtavascular microglia occur when microglia are actively colonizing the cortex

Over development, microglia undergo a dynamic process of colonization and expansion in a rostral-to-caudal gradient (Perry et al., 1985; Ashwell, 1991). Similar to previously published work (Nikodemova et al., 2015), we identified a large expansion in cortical microglia between P1 and P14, with microglia colonizing the more rostral frontal cortex region before the more caudal somatosensory cortex [Fig. 3A-C (Fig. 3B,C, bar graphs); oneway ANOVA with Dunnett's post hoc; comparison with P21 or greater, $n=4$ littermates per developmental time point, $\left.{ }^{*} p=0.0182, \quad{ }^{* *} p=0.0062, \quad{ }^{* * *} p<0.0001\right]$. Microglia-vascular association mirrored this rostral-to-caudal gradient by which microglia colonize the brain with a higher percentage of juxtavascular microglia at $\mathrm{P} 1-\mathrm{P} 5(46.3 \%$ at $\mathrm{P} 1 ; 44.4 \%$ at $\mathrm{P} 5)$ in the frontal cortex and at P5-P7 $(39.1 \%$ at $\mathrm{P} 5 ; 34.2 \%$ at $\mathrm{P} 7)$ in the more caudal somatosensory cortex (Fig. $3 B, C$, line graphs; oneway ANOVA with Dunnett's post hoc test; comparison with P21 or greater, $n=4$ littermates per developmental time point, $\left.{ }^{++}+{ }^{+} p<0.0001\right)$. Moreover, during times of active microglial colonization in both postnatal cortical regions (P1-P5 in the frontal cortex and P1-P7 in the somatosensory cortex), significantly more microglial primary processes were aligned parallel with vessels compared with older ages [Fig. 3D-G; one-way ANOVA with Dunnett's post hoc; comparison with P21 or greater, $n=4$ littermates per developmental time point, frontal cortex: ${ }^{* *} p=0.0021 \quad(\mathrm{P} 1),{ }^{* *} p=0.0033 \quad$ (P5), somatosensory 

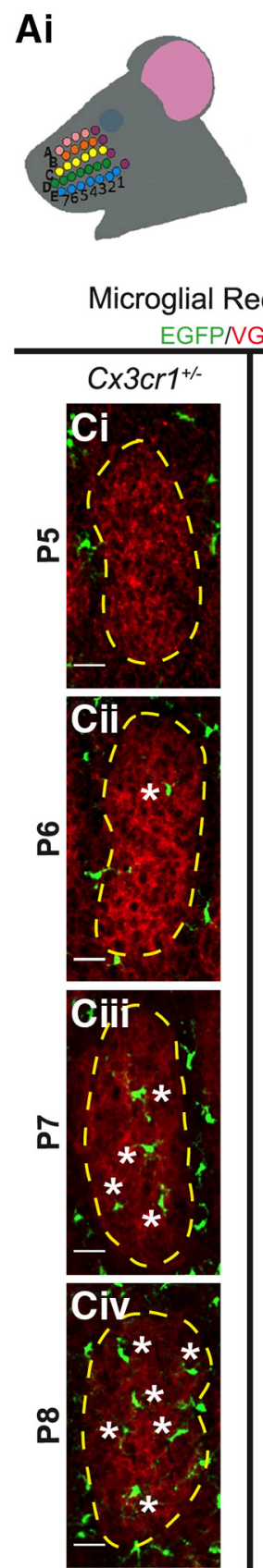

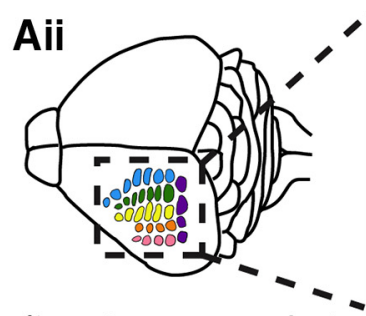

Juxtavascular Microglia EGFP/PECAM
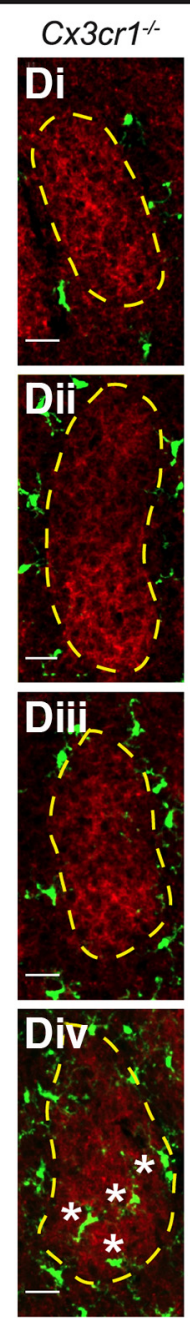
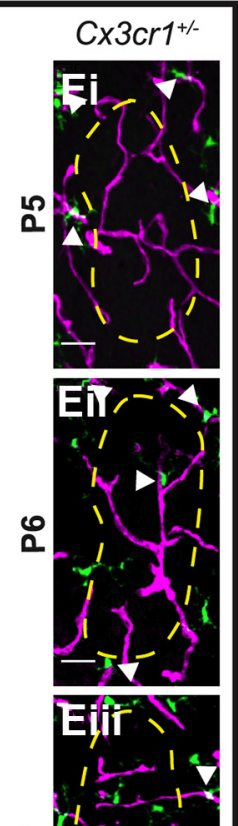

ลิ

$\frac{1}{d}$
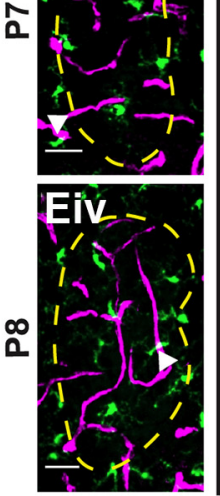

$\mathrm{Cx} \times \mathrm{cr}^{1}$
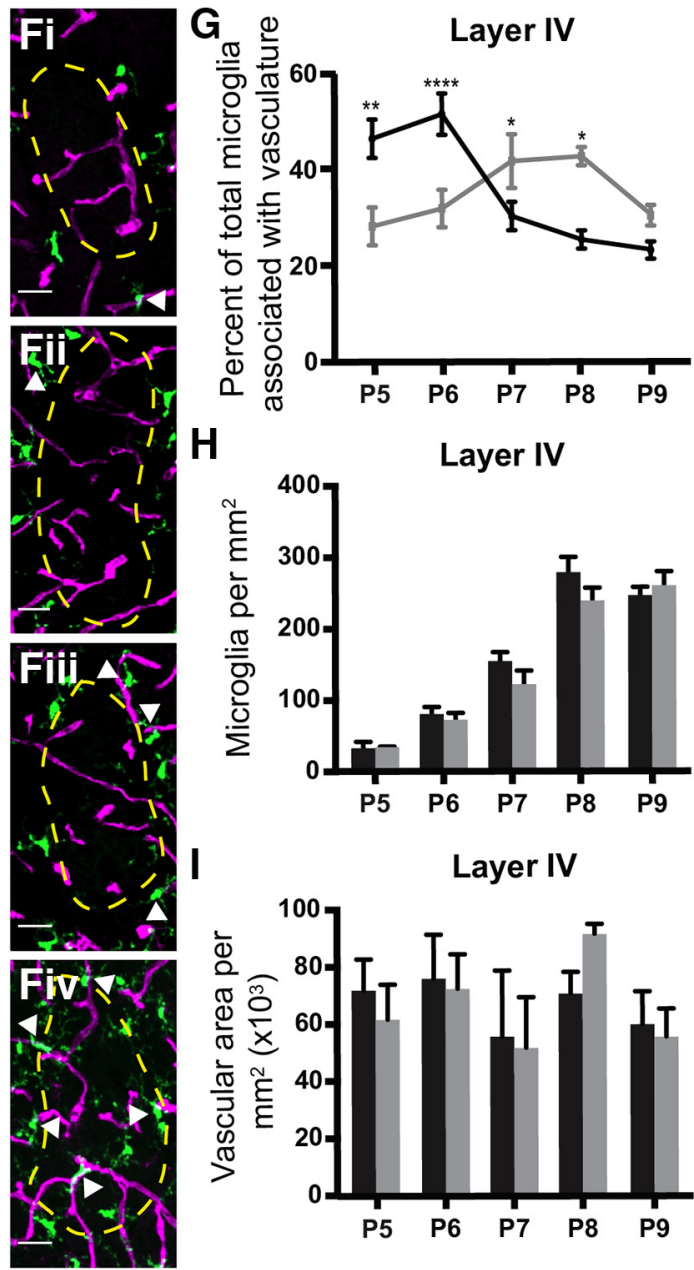

Figure 4. A high percentage of microglia associates with vasculature as they are recruited to synapses in the cortex and the timing is regulated by CX3CR1. Ai, Aii, Layer IV of the barrel cortex contains thalamocortical synapses, which form a highly precise synaptic map of the vibrissae (whiskers) on the snout. Aiii, A low-magnification representative image of a tangential section through layer IV of the barrel cortex shows that layer IV thalamocortical presynaptic terminals (red, anti-VGluT2) form discrete barrel structures corresponding to each whisker, which are separated by septa where thalamocortical terminals are largely absent. Microglia are labeled by EGFP (green) and the vasculature is labeled with anti-PECAM (gray). White box denotes a single barrel. Scale bar, $100 \mu \mathrm{m}$. B, Quantification of the number of microglia per $\mathrm{mm}^{2}$ within the barrel centers in developing $\left(\mathrm{C3}_{\mathrm{Cr}}{ }^{+/-}\right.$mice (black bars) and $\left(\mathrm{C}_{3} \mathrm{cr} 1^{-1-}\right.$ mice (gray bars). Two-way ANOVA with a Sidak's post hoc test; $n=4$ littermates per genotype per developmental time point; ${ }^{* *} p=0.0049,{ }^{* * *} p=0.0004$. Ci-Div, Representative images of quantification in $\boldsymbol{B}$. Images are zoomed in to show single barrels within tangential sections of layer IV of the barrel cortex (Aiii, white box) where microglia (green) are recruited to barrel centers in $\mathrm{CX}_{3 \mathrm{Cr}} \mathrm{C}^{+/-}$by P7 (CiCiv) and in $\mathrm{C}_{3} \mathrm{Cr}^{-1-}$ by P8 (Di-Div). Asterisks denote microglia located within barrel centers. The dotted yellow lines denote the perimeters of the VGluT2 ${ }^{+}$thalamocortical inputs (red), which define the barrels versus the septa. Scale bars, $30 \mu \mathrm{m}$. Ei-Fiv, The same representative fields of view as in Ci-Div but lacking the anti-VGluT2 channel and, instead, including the channel with anti-PECAM immunostaining (magenta) to label the vessels. Microglia are still labeled with EGFP (green). Dotted yellow lines still denote the perimeters of the VGluT2 ${ }^{+}$barrels ( $\mathbf{C}$ Div, red). Juxtavascular microglia in $\mathrm{C}_{3} \mathrm{Cr} 1^{+/-}$mice (Ei-Eiv) and $\mathrm{C} \times 3 \mathrm{Cr}^{-1-}$ mice (Fi-Fiv) are denoted by filled arrowheads. Scale bar, $30 \mu \mathrm{m}$. G, Quantification of the percentage of microglia associated with the vasculature in $\mathrm{C}_{3} \mathrm{Cr}^{+/-}$animals (black lines) and $\mathrm{C}_{3} 3 \mathrm{Cr}^{-/-}$animals (gray lines) over development in layer IV of the barrel cortex demonstrates a peak of vascular association in $\mathrm{C}_{3} \mathrm{Cr}^{+/-}$mice at P5-P6, which is delayed to P7-P8 in $\mathrm{C} \times 3 \mathrm{Cr}^{-1-}$ mice coincident with delayed microglial recruitment to barrel centers. Two-way ANOVA with a Tukey's post hoc

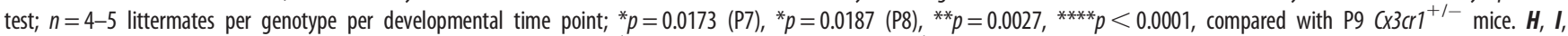
Quantification of microglial density $(\boldsymbol{H})$ and vascular density $(\boldsymbol{I})$ in $\mathrm{C} \times 3 \mathrm{Cr} 1^{+/-}$animals (black bars) and $\left(\mathrm{C}_{3} \mathrm{Cr} 1^{-1-}\right.$ animals (gray bars) over development in layer IV of the barrel cortex. Twoway ANOVA with a Sidak's post hoc test; $n=4$ littermates per genotype per developmental time point. All error bars represent \pm SEM. 
cortex: $\left.{ }^{* *} p=0.0003,{ }^{* * *} p<0.0001\right]$. This parallel juxtavascular microglial orientation along vessels is consistent with a migratory orientation.

\section{CX3CR1 regulates the timing of microglia-vascular interactions as microglia colonize synapse-dense cortical regions}

To further investigate microglia-vascular interactions in the context of colonization of the postnatal cortex, we assessed a somatosensory subregion where the pattern of microglial colonization has been well described-the barrel cortex. Layer IV of the barrel cortex contains thalamocortical synapses, which form a highly precise synaptic map of the vibrissae (whiskers) on the snout. These layer IV thalamocortical synapses form discrete barrel structures corresponding to each whisker, which are separated by septa where thalamocortical synapses are largely absent (Fig. 4A; Woolsey and Van der Loos, 1970; Welker and Woolsey, 1974). Previous work has shown that microglia first localize to the septa and then colonize these thalamocortical synapse-dense barrel centers between P6 and P7, and this process is delayed to P8-P9 in CX3CR1-deficient $\left(\mathrm{C} \times 3 \mathrm{cr}^{-1-}\right)$ mice (Hoshiko et al., 2012). This delay in recruitment in $\mathrm{Cx} 3 \mathrm{Crl}^{-1-}$ mice is concomitant with a delay in synapse maturation. However, it was unclear how CX3CR1 was regulating the timing of microglial recruitment to synapses in the barrel cortex. To identify barrels, we labeled thalamocortical presynaptic terminals with an antibody against VGluT2. Microglia were labeled with transgenic expression of EGFP in either $C \times 3 \mathrm{crl}^{+/-}$mice $\left(\mathrm{C} \times 3 \mathrm{cr} 1^{\mathrm{EGFP} /+}\right)$ or $\mathrm{C} \times 3 \mathrm{cr} 1^{-/-}$mice $\left(C \times 3 c r 1^{E G F P / E G F P}\right)$. The vasculature was labeled with antiPECAM. Similar to previous work (Hoshiko et al., 2012), microglia infiltrated thalamocortical synapse-dense barrel centers (Fig. 4C-F, outlined with a yellow dotted line) from the septa by $\mathrm{P} 6-\mathrm{P} 7$ in $\mathrm{C} \times 3 \mathrm{cr} 1^{+/-}$mice, and this process was delayed by $1 \mathrm{~d}$ in $C \times 3 \mathrm{cr} 1^{-1-}$ mice (Fig. $4 B-D$; two-way ANOVA with a Sidak's post hoc; $n=4$ littermates per genotype per developmental time point; ${ }^{* *} p=0.0049$, $\left.{ }^{* *} p=0.0004\right)$. Strikingly, just before entering barrel centers at P5-P6 in $\mathrm{C} \times 3 \mathrm{crl}^{+}{ }^{-}$mice, a higher percentage of microglia were juxtavascular (Fig. 4E,G, arrowheads; two-way ANOVA with a Tukey's post hoc test; $n=4-5$ littermates per genotype per developmental time point; ${ }^{* *} p=0.0027,{ }^{* * * *} p<0.0001$, compared with P9 $\mathrm{C} x 3 \mathrm{CrI}^{+/-}$). Further, this microglia-vascular association was delayed by $1 \mathrm{~d}$ in $C \times 3 \mathrm{cr} 1^{-1-}$ mice [Fig. $4 F, G$; two-way ANOVA with a Tukey's post hoc test; $n=4-5$ littermates per genotype per developmental time point; ${ }^{\star} p=0.0173$ (P7), ${ }^{\star} p=0.0187$ (P8), compared with P9 Cx3cr1 ${ }^{+/-}$mice], which is consistent with the delay in microglial migration into barrel centers in these mice (Fig. $4 B$ ). In both genotypes, the percentage of juxtavascular microglia decreased once the microglia began to colonize the thalamocortical synapse-dense barrel centers, at P7 in $\mathrm{C} x 3 \mathrm{cr} 1^{+/-}$mice and at P8 in $\mathrm{C} \times 3 \mathrm{cr} 1^{-1-}$ mice (Fig. $4 E-G$ ). These changes in microglia-vascular interactions were independent of any changes in total microglial or vascular density in layer IV (Fig. 4H,I; two-way ANOVA with a Sidak's post hoc test; $n=4$ littermates per genotype per developmental time point), but rather specific to microglial distribution between the septa and barrels. These data are consistent with a model by which microglia use the vasculature to colonize synapse-dense cortical regions at the appropriate developmental timing. They further suggest that CX3CR1 signaling modulates the timing of microglial-vascular interactions during colonization.
Juxtavascular microglia migrate along the vasculature as they colonize the developing brain and are stationary in adulthood

With data demonstrating that high percentages of microglia are juxtavascular when they are actively colonizing the brain with processes aligned parallel to the vessel, we next performed live imaging to assess migration. As the early postnatal cortex is challenging to image in vivo, we performed our initial analyses in acute cortical slices. Acute slices of somatosensory cortex were prepared from early postnatal (P7) and adult $(p \geq 120)$ $\mathrm{C} \times 3 \mathrm{cr} 1^{E G F P /+}$ mice, which were given a retro-orbital injection of Texas Red-labeled dextran to label blood vessels before slice preparation. We then imaged microglia every 5 min over $6 \mathrm{~h}$ at both ages (Fig. 5A). Live imaging at P7 revealed significant juxtavascular microglial soma movement along blood vessels in the somatosensory cortex compared with vascular-unassociated microglia at P7 (Fig. 5B,D, Movies 3, 4, 5). Specifically, $28.6 \%$ of juxtavascular microglia somas moved at a rate of $3-5 \mu \mathrm{m} / \mathrm{h}$ and another $26.1 \%$ moved at a rate of $5-7.5 \mu \mathrm{m} / \mathrm{h}$ [Fig. $5 D$; two-way ANOVA with a Sidak's post hoc test; $n=4$ mice per time point; ${ }^{* *} p=0.0081$ (stationary), ${ }^{* *} p=0.0013(0-3 \mu \mathrm{m} / \mathrm{h}),{ }^{* *} p=0.0015$ $\left.(5-7.5 \mu \mathrm{m} / \mathrm{h}),{ }^{* *} p=0.0005(3-5 \mu \mathrm{m} / \mathrm{h})\right]$. In comparison, only $9.3 \%$ and $6.8 \%$ of vascular-unassociated microglia at the same age moved at $3-5$ and $5-7.5 \mu \mathrm{m} / \mathrm{h}$, respectively. We further found that when we assessed just the motile soma at P7, significantly more juxtavascular microglia somas traveled $>20$ $\mu \mathrm{m}$ (30.9\% traveled $20-30 \mu \mathrm{m}$, and $23.6 \%$ traveled $30-45$ $\mu \mathrm{m})$ over $6 \mathrm{~h}$ compared with vascular-unassociated microglia (7.5\% and $6.8 \%$ traveled $20-30$ and $30-45 \mu \mathrm{m}$, respectively; Fig. 5E; two-way ANOVA with a Sidak's post hoc test; $n=4$ mice; $\left.{ }^{* *} p=0.0041,{ }^{* * * *} p<0.0001\right)$. Importantly, the juxtavascular microglia soma velocities and distances traveled are consistent with the rate and distances at which microglia migrate to barrel centers within the somatosensory cortex in vivo where the distance between the septa and barrel center is $\sim 80 \mu \mathrm{m}$, and it takes $\sim 24 \mathrm{~h}$ for microglia to reach the barrel center from the septa. Demonstrating directional motility and suggesting migration along the vessel, $84.1 \%$ of these postnatal juxtavascular microglia had a motility trajectory of $\leq 15^{\circ}$ along the blood vessel (Fig. 5F; unpaired Student's $t$ test; $n=4$ mice per time point; $\left.{ }^{* * *} p<0.0001\right)$. Together, these data demonstrate directional migration of juxtavascular microglia at distances and speeds consistent with colonization of the cortex.

Interestingly, this migratory behavior along the vasculature was developmentally regulated and juxtavascular microglia in adult slices were largely stationary (Fig. 5C,D, Movie 6). We further confirmed the stationary phenotype of juxtavascular microglia in the adult cortex by in vivo two-photon live imaging in CX3 $\mathrm{crl}^{E G F P /+}$ mice. Cranial windows were implanted over the visual cortex, which was most conducive to our headposts necessary for stabilizing the head in awake, behaving mice during imaging. We have found similar microglia-vascular interactions by static confocal imaging in the visual cortex (data not shown). Mice were given a retro-orbital injection of Texas Red-labeled dextran to label blood vessels before imaging and juxtavascular microglia were imaged every $5 \mathrm{~min}$ over the course of $2 \mathrm{~h}$ through the cranial window (Fig. $5 G$ ). As observed in acute cortical slices, $100 \%$ of juxtavascular and vascular-unassociated microglia were stationary (Fig. $5 \mathrm{H}$, Movie 7 , unpaired Student's $t$ test; $n=3$ mice per developmental time point). To further understand long-term dynamics, we imaged juxtavascular microglia in vivo over the course of 6 weeks (Fig. 5I) in awake, behaving mice. 
A

CX3Cr1EGFP/+

$P 7$ and $P \geq 120$

Retro-orbital

Dextran Texas

Red
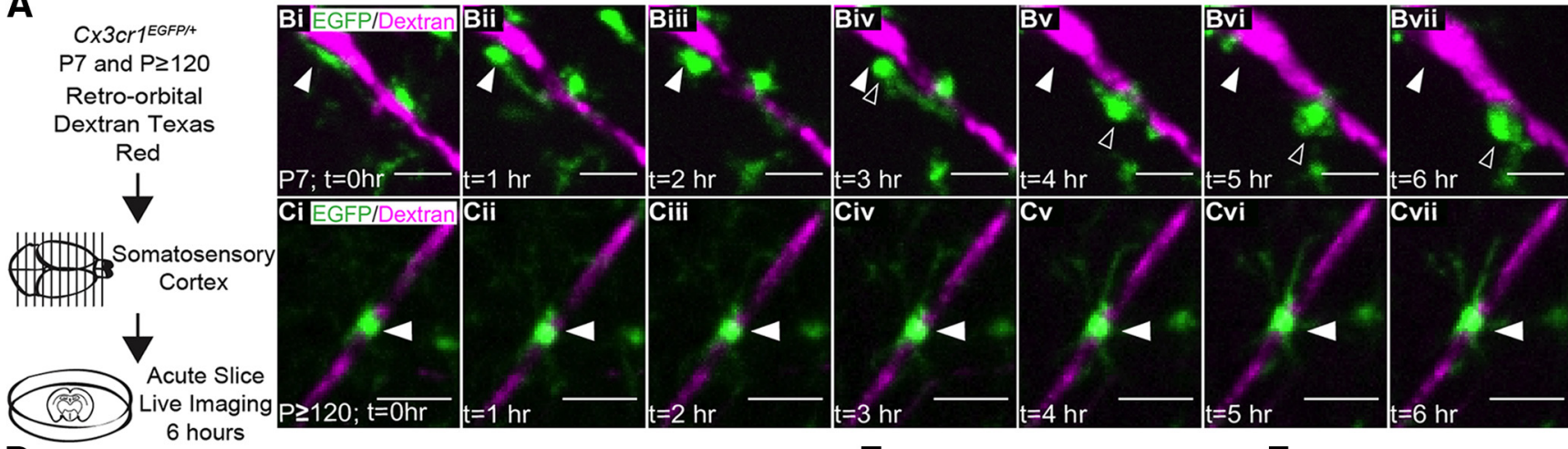

D
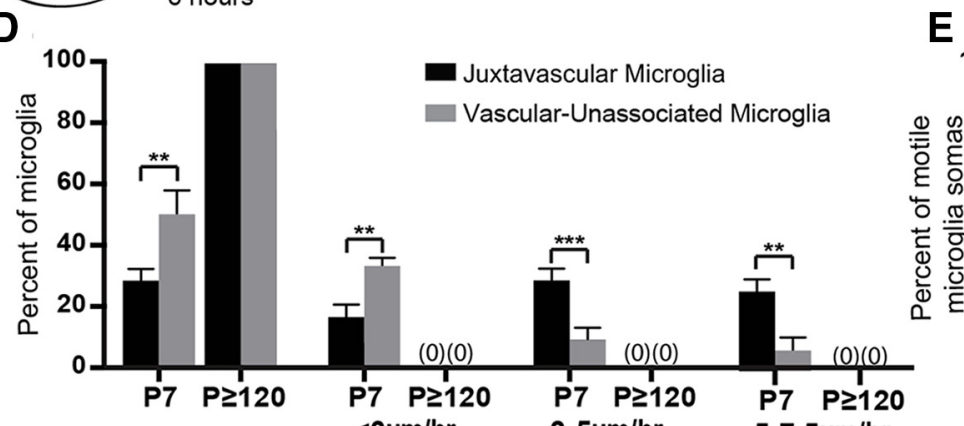

E

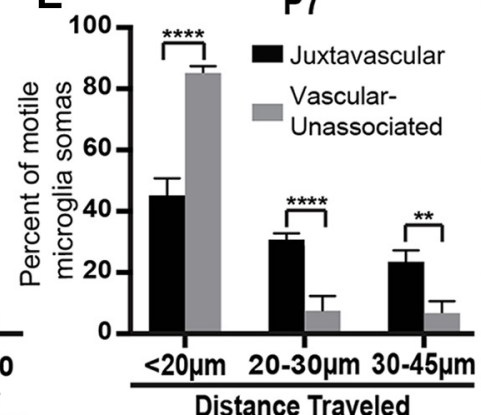

$F_{0}$

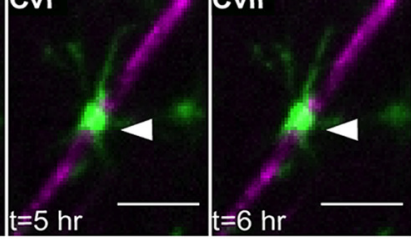

nascular-Unassociated Microglia
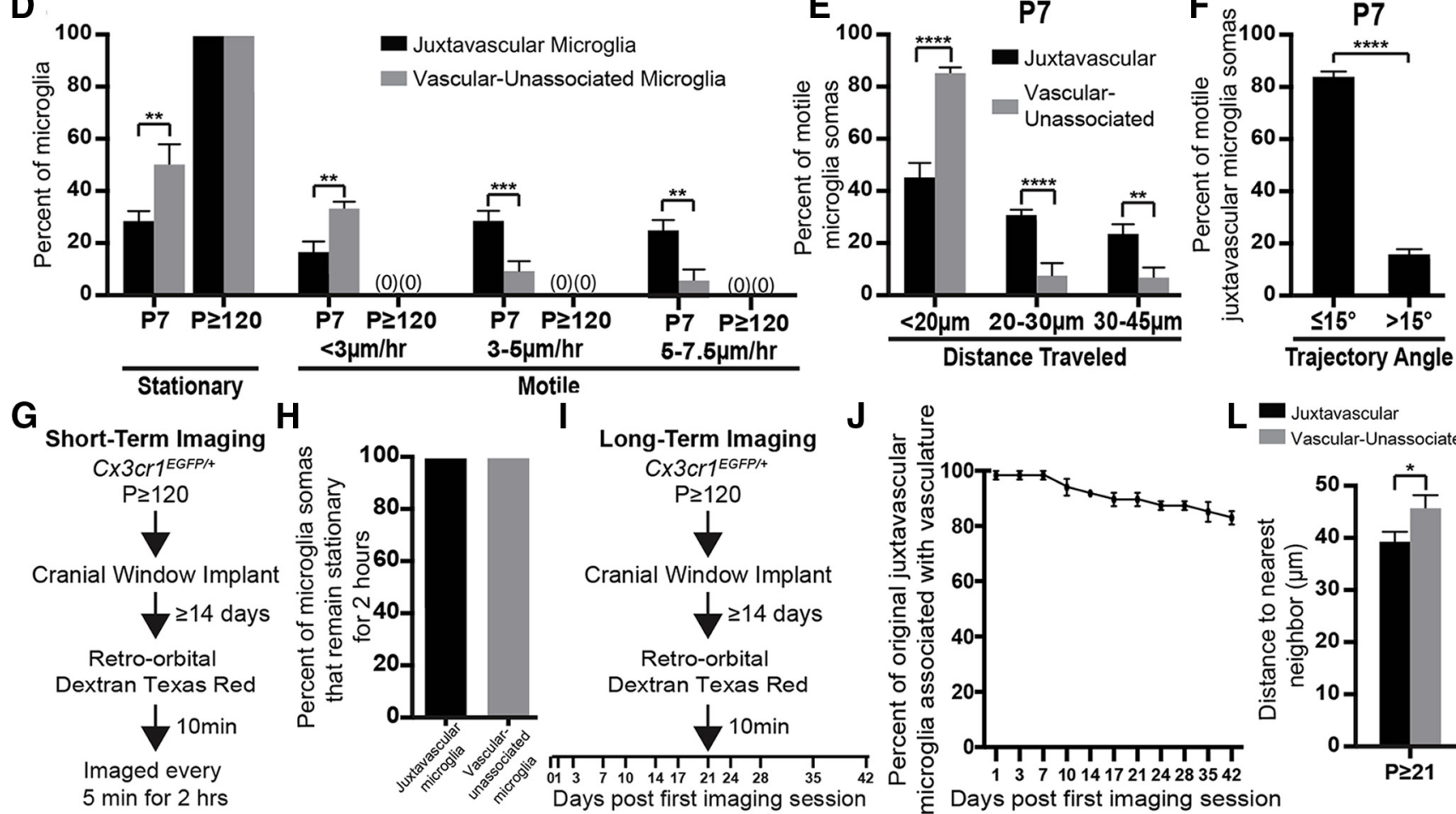

Distance Traveled

Trajectory Angle
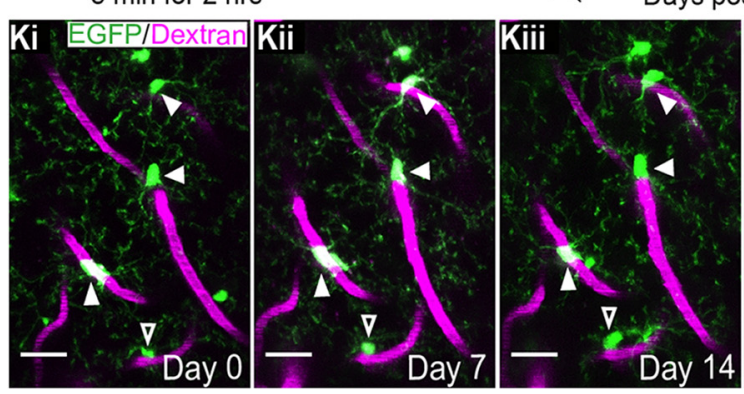

I

Long-Term Imaging

J

CX3Cr1EGFP/+

$\mathrm{P} \geq 120$

$\checkmark$

Cranial Window Implant

$\checkmark \geq 14$ days

Retro-orbital

Dextran Texas Red

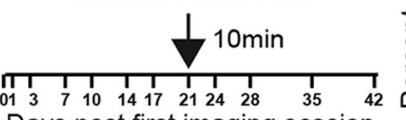

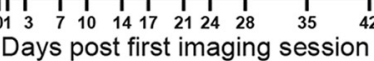
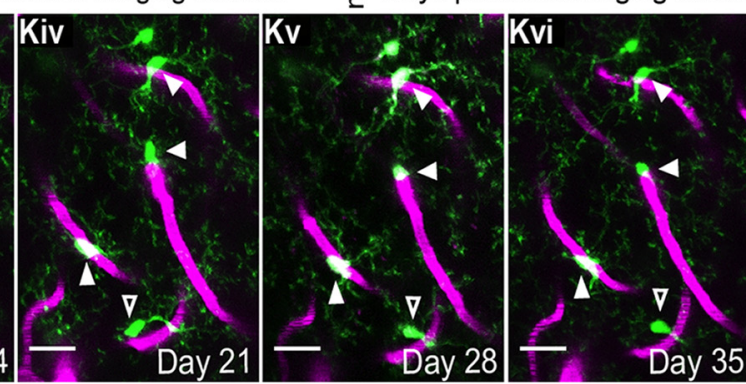

L Juxtavascular

Vascular-Unassociated

Figure 5. Juxtavascular microglia migrate along blood vessels as they colonize the developing brain and are largely stationary in adulthood. $\boldsymbol{A}$, A schematic of the live-imaging experiment. $C \times 3 \mathrm{Cr}^{\text {EGFP/+ }}$ mice received a retro-orbital injection of Texas red-labeled dextran to label the vasculature 10 min before acute slice preparation. Coronal somatosensory cortices were then imaged every $5 \mathrm{~min}$ over $6 \mathrm{~h}$ immediately following slice preparation. $\mathbf{B i}$-Cvii, Representative fluorescent images from a $6 \mathrm{~h}$ live-imaging session from a P7 (Bi-Bvii) and P120 or greater (Ci-Cvii) slice. Filled arrowheads indicate microglial soma position at $t=0$. Unfilled arrowheads indicate the location of the same microglial soma at $0 \mathrm{~h}$ (Bi, Ci), $1 \mathrm{~h}$ (Bii, Cii), $2 \mathrm{~h}$ (Biii, Ciii), $3 \mathrm{~h}$ (Biv, Civ), 4 h (Bv, Cv), 5 h (Bvi, Cvi), and 6 h (Bvii, Cvii; Movies 3, 4, 5, 6). Scale bars, $30 \mu \mathrm{m}$. D, Quantification of juxtavascular (black bars) and vascular-unassociated (gray bars) microglia soma motility speed/velocity. Two-way ANOVA with a Sidak's post hoc test; $n=4$ mice per time point; ${ }^{* *} p=0.0081\left(\right.$ stationary) ${ }^{* *} p=0.0013(0-3 \mu \mathrm{m} / \mathrm{h})$, ${ }^{* *} p=0.0015(5-7.5 \mu \mathrm{m} / \mathrm{h})$, ${ }^{* * *} p=0.0005(3-5 \mu \mathrm{m} / \mathrm{h}) . \boldsymbol{E}$, Quantification of the distance traveled of juxtavascular (black bars) and vascular-unassociated (gray bars) microglia somas in the P7 somatosensory cortex. Twoway ANOVA with a Sidak's post hoc test; $n=4$ mice; ${ }^{* *} p=0.0041,{ }^{* * * *} p<0.0001$. $\boldsymbol{F}$, Quantification of migratory juxtavascular microglia trajectory angles in the P7 somatosensory cortex. Unpaired Student's $t$ test; $n=4$ mice per time point; ${ }^{* * *} p<0.0001$. $\boldsymbol{G}$, A schematic of a short-term two-photon live-imaging experiment in adult cortex of awake, behaving mice. $\mathrm{CX3}_{\mathrm{Cr}} 7^{\text {EGFP/+ }}$ mice received a retro-orbital injection of Texas Red-labeled dextran to label the vasculature 10 min before each imaging session. EGFP ${ }^{+}$juxtavascular microglia were then imaged every $5 \mathrm{~min}$ for $2 \mathrm{~h}$ through a cranial window (Movie 7). $\boldsymbol{H}$, Quantification of the percentage of juxtavascular (black bars) and vascular-unassociated (gray bars) microglia that remain stationary for $2 \mathrm{~h}$. Unpaired Student's $t$ test; $n=3$ mice per developmental time point. I, A schematic of the long-term two-photon live-imaging experiment in adult visual cortex. $C \times 3 c r 7^{\text {EGFP/+ }}$ mice received a retro-orbital injection of Texas Red-labeled dextran to visualize the vasculature 10 min before each imaging session. EGFP ${ }^{+}$juxtavascular microglia were then imaged for 6 weeks through a cranial window. J, Quantification of the percentage of juxtavascular microglia on vessels on day 0 that remain on vessels through 6 weeks of imaging. Data are representative of $n=3$ mice. $\boldsymbol{K i}$-Kvii, Representative fluorescent images acquired during a 6 -week live-imaging session from a single mouse. Filled arrowheads indicate juxtavascular microglia that remain on 


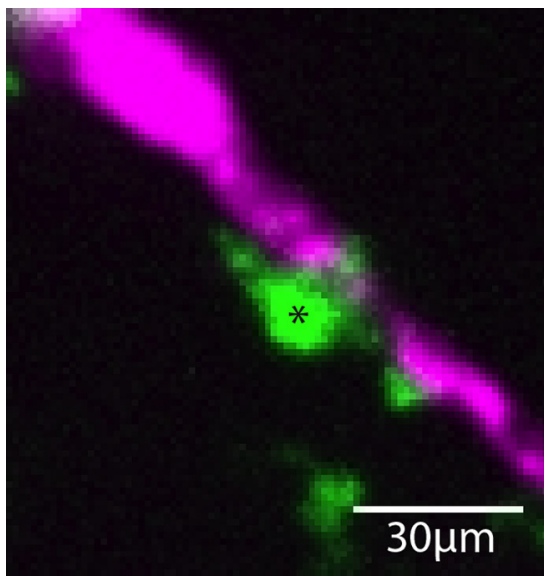

Movie 3. Juxtavascular microglial migration in the early postnatal somatosensory cortex. Representative live imaging of juxtavascular microglia (green, EGFP) migrating on vessels (magenta, dextran) in the P7 somatosensory cortex. $\left(x 3 \mathrm{cr} 7^{\text {EGFP/+ }}\right.$ mice received a retro-orbital injection of Texas red-labeled dextran to label the vasculature 10 min prior to slice preparation. Coronal somatosensory cortices were imaged every 5 min over $6 \mathrm{~h}$ immediately following slice preparation. Asterisk in still image denotes the microglia that were tracked for quantification. [View online]

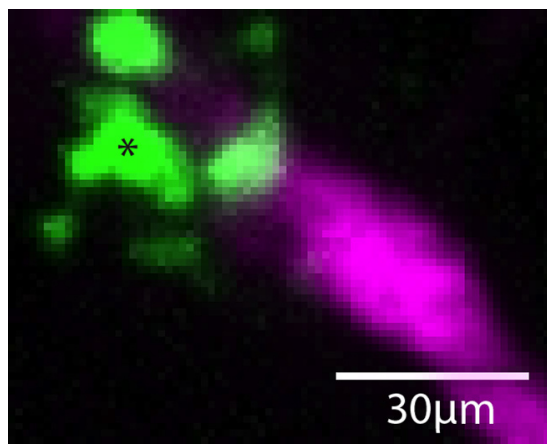

Movie 4. Juxtavascular microglial migration in the early postnatal somatosensory cortex. A second representative live imaging of juxtavascular microglia (green, EGFP) migrating on vessels (magenta, dextran) in the P7 somatosensory cortex. $C x 3 \mathrm{Cr}^{\mathrm{EGFP} /+}$ mice received a retro-orbital injection of Texas red-labeled dextran to label the vasculature $10 \mathrm{~min}$ prior to slice preparation. Coronal somatosensory cortices were imaged every 5 min over $6 \mathrm{~h}$ immediately following slice preparation. Asterisk in still image denotes the microglia that were tracked for quantification. [View online]

We identified that $82.9 \%$ of juxtavascular microglia present on day 0 of imaging remained near the vasculature 6 weeks later (Fig. 5J,K). Using static confocal imaging, we also found that juxtavascular microglia were less evenly distributed to their nearest neighbor compared with vascular-unassociated microglia, suggesting a preferential association with vessels in the adult versus simply a consequence of tiling (Fig. $5 L$; paired Student's $t$ test; $n=4$ littermates, ${ }^{*} p=0.0239$ ). Together, these data demonstrate that juxtavascular microglia in the postnatal cortex are highly migratory compared with nonvascular associated microglia. In contrast, juxtavascular microglia in adulthood have a distribution that is less tiled compared with other parenchymal microglia,

$\leftarrow$

vessels for 6 weeks. Unfilled arrowhead, A juxtavascular microglia that changes position, but remains on the vasculature, for over 6 weeks. $L$, Quantification of the nearest neighbor distance between juxtavascular and vascular-unassociated microglia in static confocal images. Paired Student's $t$ test; $n=4$ littermates, ${ }^{*} p=0.0239$. All error bars represent \pm SEM.

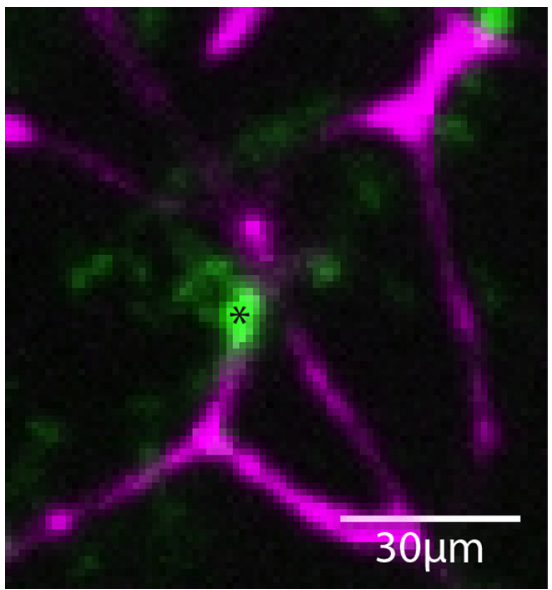

Movie 5. Juxtavascular microglial migration in the early postnatal somatosensory cortex. A third representative live imaging of juxtavascular microglia (green, EGFP) migrating on vessels (magenta, dextran) in the P7 somatosensory cortex. $C \times 3 \mathrm{Cr}^{\mathrm{EGFP} /+}$ mice received a retroorbital injection of Texas red-labeled dextran to label the vasculature $10 \mathrm{~min}$ prior to slice preparation. Coronal somatosensory cortices were imaged every 5 min over $6 \mathrm{~h}$ immediately following slice preparation. Asterisk in still image denotes the microglia that were tracked for quantification. [View online]

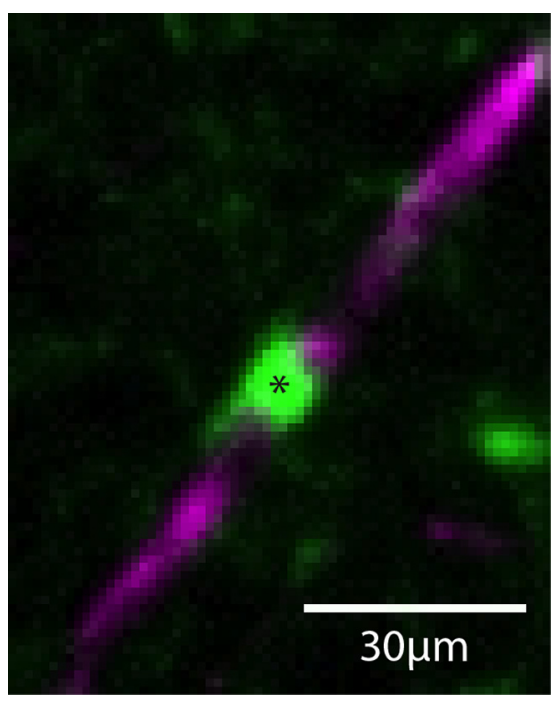

Movie 6. Juxtavascular microglial migration in the adult somatosensory cortex. Representative live imaging of juxtavascular microglia (green, EGFP) stationary on vessels (magenta, dextran) in the P120 or greater somatosensory cortex. $\left(x 3 \mathrm{Cr}^{\mathrm{EGFP} /+}\right.$ mice received a retro-orbital injection of Texas red-labeled dextran to label the vasculature $10 \mathrm{~min}$ prior to slice preparation. Coronal somatosensory cortices were imaged every 5 min over $6 \mathrm{~h}$ immediately following slice preparation. Asterisk in still image denotes the microglia that was tracked for quantification. [View online]

and they are largely stationary, which suggests the establishment of a niche for juxtavascular microglia in the adult brain.

\section{Microglia associate with the vasculature in areas lacking full astrocyte endfoot coverage}

Our data demonstrate a strong microglial association and migration along the developing postnatal cortical vasculature. One possible mechanism regulating these developmental changes in juxtavascular microglia is the changing cellular composition of the NVU over the course of development. The NVU begins to form during embryonic development, when pericytes associate with endothelial cells. Later in postnatal development, astrocytes 


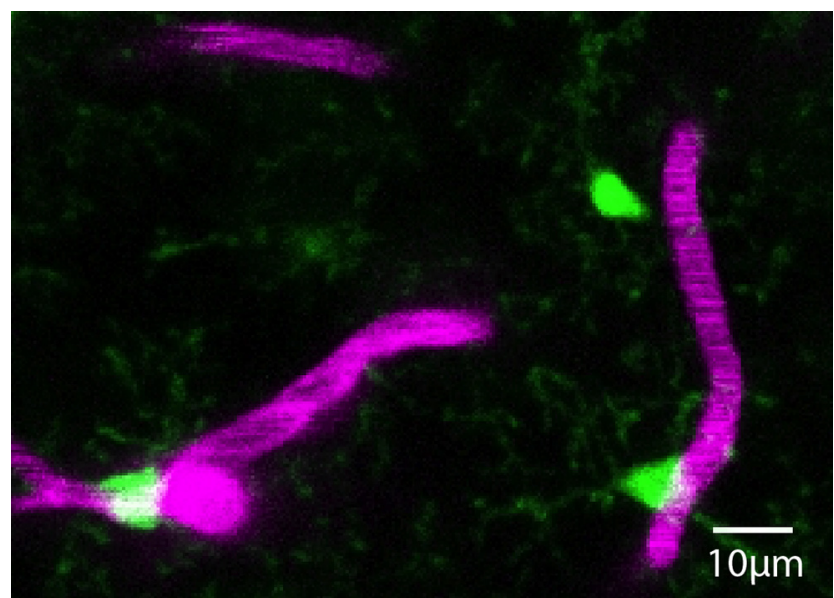

Movie 7. Two-photon in vivo live imaging of juxtavascular microglia in the adult cortex. Representative two-photon in vivo live imaging of juxtavascular microglia (green, EGFP) stationary on blood vessels (magenta, dextran) over $2 \mathrm{~h}$ in vivo in the adult cortex. $\left(\times 3 \mathrm{Cr} 7^{\text {EGFP/+ }}\right.$ mice received a retro-orbital injection of Texas Red-labeled dextran to label the vasculature 10 min prior to each imaging session. $\mathrm{EGFP}^{+}$juxtavascular microglia were then imaged every $5 \mathrm{~min}$ for $2 \mathrm{~h}$ through a cranial window in awake, behaving mice. [View online]

are born and begin wrapping their endfeet around vessels until the vast majority of the vasculature is ensheathed by astrocyte endfeet by adulthood (Daneman et al., 2010; Bayraktar et al., 2014; Schiweck et al., 2018). As previously described (Daneman et al., 2010), the territory of $\mathrm{AQP}^{+}$astrocyte endfeet on $\operatorname{PDGFR} \beta^{+}$capillaries was low in the early postnatal cortex and then expanded over the first postnatal week [Fig. 6A-D (bar graph in Fig. 6D); one-way ANOVA with Dunnett's post hoc test; comparison with $\mathrm{P} 21$ or greater, $n=3$ littermates per developmental time point, $\left.{ }^{* * *} p=0.0005,{ }^{* * *} p<0.0001\right]$. In more mature animals (P21 or greater), astrocytic endfeet covered $\sim 85 \%$ of vessels in the frontal cortex. Intriguingly, this developmental timing of astrocyte endfoot coverage mirrored the developmental shift in the percentage of juxtavascular microglia in the cortex (Fig. 6D, line graph; one-way ANOVA with Dunnett's post hoc test; comparison with $\mathrm{P} 21$ or greater, $n=4$ littermates per developmental time point, $\left.{ }^{++++} p<0.0001\right)$. That is, as the percentage of juxtavascular microglia decreased, astrocyte endfoot coverage increased. This astrocyte coverage also correlated with the timing of decreased microglial motility along the vessels (Fig. 5). We next assessed microglia-astrocyte endfoot interactions by confocal microscopy and 3D surface rendering at all ages and found that microglia only associated with the vasculature in areas either completely void of astrocyte endfeet or in areas where vessels were not fully covered by the endfeet (Fig. $6 A-C$, white arrowheads, Fig. $6 E$, Movies $8,9,10)$. We also found microglia occupied $4-9 \%$ of the vessel area, depending on postnatal age, and there were parts of the vessel that lacked astrocyte endfeet that also lacked microglia (Fig. 6F).

Given that cells of the NVU are nanometers apart from each other, we confirmed these results with expansion microscopy (ExM) (Fig. 6G,H), structured illumination microscopy (SIM) (Fig. 6I,J), and electron microscopy (EM) (Fig. 7). Microglia were identified by EM based on characteristic microglial morphologies. Microglia nuclei tend to be half-moon shaped or long and thin with electron dense heterochromatin around the edge of the nucleus. Microglia were further distinguished by EM from perivascular macrophages by having processes emanating from the soma. Serial sectioning and 3D reconstruction of a representative cell captured by EM from each age confirmed that juxtavascular microglia contacted the basal lamina in vascular areas without full astrocyte endfoot coverage at all ages (Fig. 7C, Movies 11, 12). Similar to light microscopy (Fig. $1 H$ ), the microglial surface area contacting the vessel decreased in older animals [Fig. 7D; unpaired Student's $t$ test; $n=29$ cells (P5) and $n=11$ cells (P56); $\left.{ }^{\star} p=0.0155\right]$, and there were areas of the vasculature that were not fully ensheathed by astrocytes by EM, which were not contacted by microglia. Together, these data demonstrate that juxtavascular microglia contact the vascular basal lamina and associate with the vasculature in areas lacking full coverage by astrocyte endfeet. The data raise the intriguing possibility that the lack of astrocyte endfeet in early postnatal development provides a permissive environment for juxtavascular microglial association with and migration along the vasculature as they colonize the brain.

\section{Discussion}

This study provides the first extensive analysis of juxtavascular microglia in the healthy developing and adult brain. We discovered that a high percentage of juxtavascular microglia is associated with large capillaries in the early postnatal mouse cortex. Similar microglia-vascular association was observed in the developing human brain. Live imaging revealed that juxtavascular microglia are migratory along the vasculature during the peak of microglial colonization of the postnatal cortex and become stationary by adulthood. In addition, microglia are highly associated with the vasculature during development as they are being recruited to synapse-dense rich cortical regions and the timing of these interactions is regulated by CX3CR1. Last, we provide evidence that microglia preferentially associate and contact the vasculature at all ages in areas lacking full astrocyte endfoot coverage and that the expansion of astrocytic endfeet along blood vessels coincides with a decrease in microglia migration along vessels. Together, these data suggest that microglia are using the vasculature to migrate and colonize the cortex and that the timing of this vascular association is regulated by CX3CR1. Our data further support a mechanism in which microglial migration along the vasculature during development ceases and juxtavascular microglia become stationary as astrocyte endfeet mature.

\section{A possible role for the vasculature in regulating microglial colonization}

Microglia are born as primitive macrophages in the embryonic yolk sac and enter the neuroepithelium at E9.5 by crossing the pial surface and lateral ventricles (Navascués et al., 2000; Ginhoux et al., 2010; Swinnen et al., 2013). Microglia then migrate and proliferate through the brain parenchyma in a rostral-to-caudal gradient to colonize the embryonic brain (Perry et al., 1985; Ashwell, 1991; Sorokin et al., 1992; Alliot et al., 1999; Navascués et al., 2000; Swinnen et al., 2013). Signaling mechanisms have been identified to regulate initial microglial infiltration into the brain parenchyma, such as matrix metalloproteinases, stromal cell-derived factor 1, and Cxcl12/Cxcr4 signaling (Ginhoux et al., 2010; Arnò et al., 2014; Ueno and Yamashita, 2014). However, far less is known about the mechanisms regulating microglial localization to the appropriate brain regions once they reach the parenchyma, particularly during postnatal development. Previous work has shown that microglia can migrate along the vasculature in acute embryonic brain slices and brain slices prepared from postnatal mice in an injury context (Grossmann et al., 2002; Smolders et al., 2017). In addition, other work has shown that oligodendrocyte precursor cells require the vasculature as a 

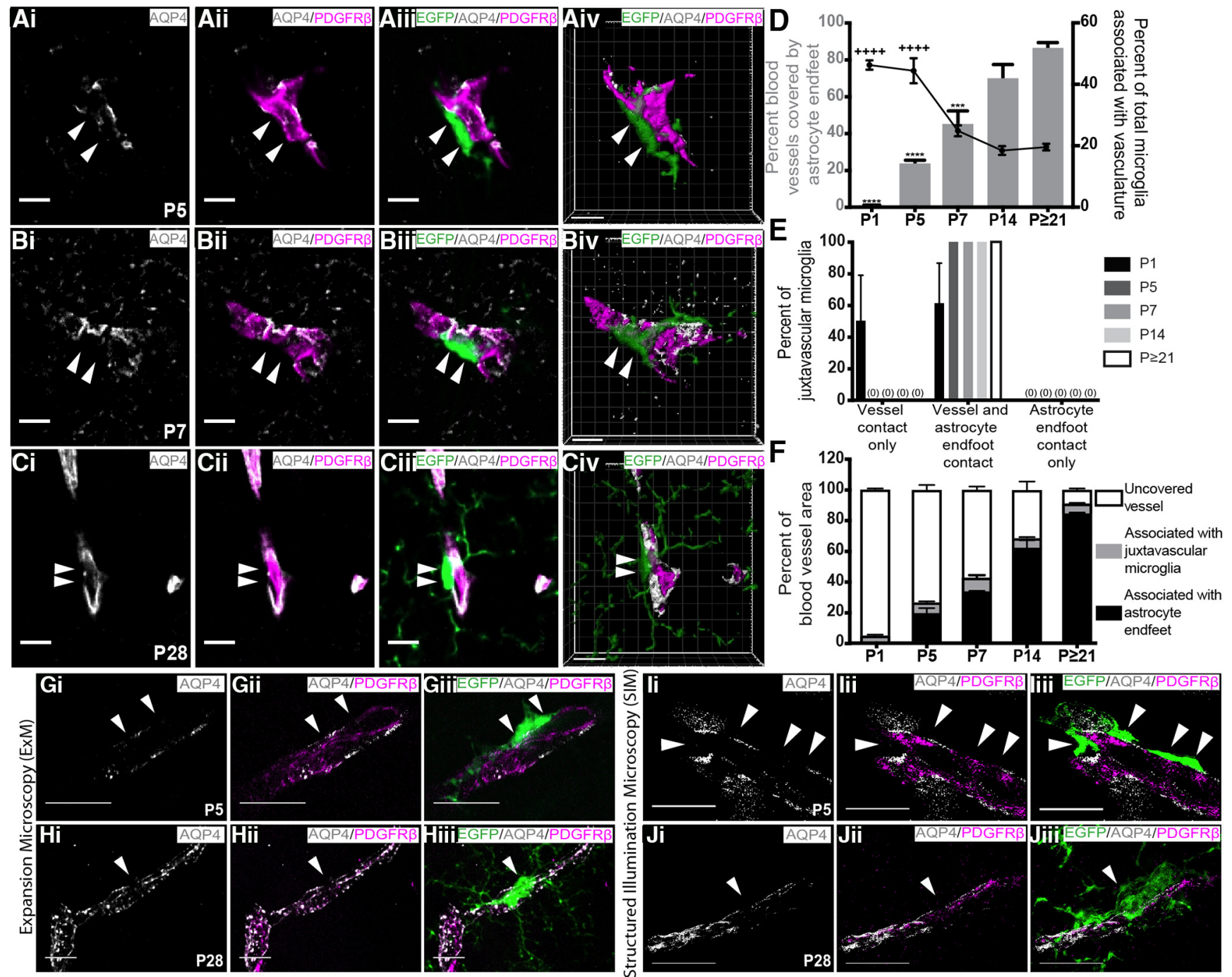

Figure 6. Juxtavascular microglia associate with the cortical vasculature in areas lacking full astrocytic endfoot coverage. Ai-Civ, Representative single-optical plane images and 3D rendering (Aiv-Civ; Movies 8, 9, 10) of juxtavascular microglia (green, EGFP) and blood vessels (magenta, anti-PDGFR $\beta$ ) in areas void of astrocytic endfoot labeling (gray, anti-AQP4) in the frontal cortex at P5 (Ai-Aiv), P7 (Bi-Biv), and P28 (Ci-Civ). Filled arrowheads denote vascular areas that lack astrocyte endfeet where juxtavascular microglia are associated with the vessel. Scale bars, $10 \mu \mathrm{m}$. D, Left, $y$-axis, Gray bars: quantification of the percentage of blood vessels covered by astrocyte endfeet over development in the frontal cortex. One-way ANOVA with Dunnett's post hoc test; comparison with P21 or greater, $n=3$ littermates per developmental time point, ${ }^{* * *} p=0.0005,{ }^{* * * *} p<0.0001$. Right, $y$-axis, Black line: the percentage of the total microglia population that are juxtavascular over development in the frontal cortex (data are the same as presented in Fig. 1G). One-way AN0VA with Dunnett's post hoc test; comparison with P21 or greater, $n=4$ littermates per developmental time point, $++++p<0.0001$. $\boldsymbol{E}$, Quantification of the percentage of juxtavascular microglia associated with vessels only, vessels and astrocyte endfeet (representative images in Aiv-Civ), and astrocyte endfeet only from 3D-rendered images. $\boldsymbol{F}$, Quantification of the percentage of blood vessel area associated with astrocyte endfeet (black bars), juxtavascular microglia (gray bars), or uncovered vessels (white bars) in the frontal cortex over development. Gi-Jiii, Representative ExM (Gi-Hiii) and SIM (Ii-Jiii) images of juxtavascular microglia (green, EGFP) in vascular areas lacking anti-AQP4 (gray) astrocytic endfoot labeling (filled arrowheads) in the P5 (Gi-liii) and P28 (Hi-Jiii) frontal cortex. Scale bars, $10 \mu$ m. All error bars represent \pm SEM.

physical substrate for migration (Tsai et al., 2016). Similar findings have been identified for neural stem cells where the timing of astrocyte endfeet to the vessels has also been implicated (Bovetti et al., 2007; Whitman et al., 2009; Fujioka et al., 2019). We have identified that microglia are highly associated with vasculature during the peak of microglial colonization and recruitment to synapses. Furthermore, these vascularassociated microglia are migratory along blood vessels during early postnatal development and later become stationary once microglial colonization is complete. We also show in $\mathrm{C} \times 3 \mathrm{crl}^{-1-}$ mice with known delays in microglial colonization of synapse-dense cortical regions that there are concomitant delays in microglial association with the vasculature. As we have observed no significant expression of $\mathrm{Cx3}$ cll (the CX3CR1 ligand) by vascular cells (Gunner et al., 2019), and a subset of microglia still associate with the vasculature in $\mathrm{C} \times 3 \mathrm{Cr}^{-1-}$ mice, this delay in microglial vascular association in $\mathrm{C} \times 3 \mathrm{crl}^{-1-}$ mice is most likely because of disruptions in chemokine gradient signaling from neuronal sources of CX3CL1 versus a direct effect of vascular adhesion. This would suggest that microglia receive directional cues from surrounding cells and use the vasculature as a substrate to migrate toward those cues. The timing of this migration along the vasculature is regulated by CX3CR1. As $\mathrm{C} x 3 \mathrm{cr} 1^{-1-}$ mice have delays in synapse maturation and pruning and, long term, have behavioral deficits consistent with an autism-like phenotype, it suggests that these microglia-vascular associations in development have long-term consequences (Paolicelli et al., 2011; Hoshiko et al., 2012; Zhan et al., 2014). The vascular cues regulating microglial adhesion and migration in the healthy CNS 


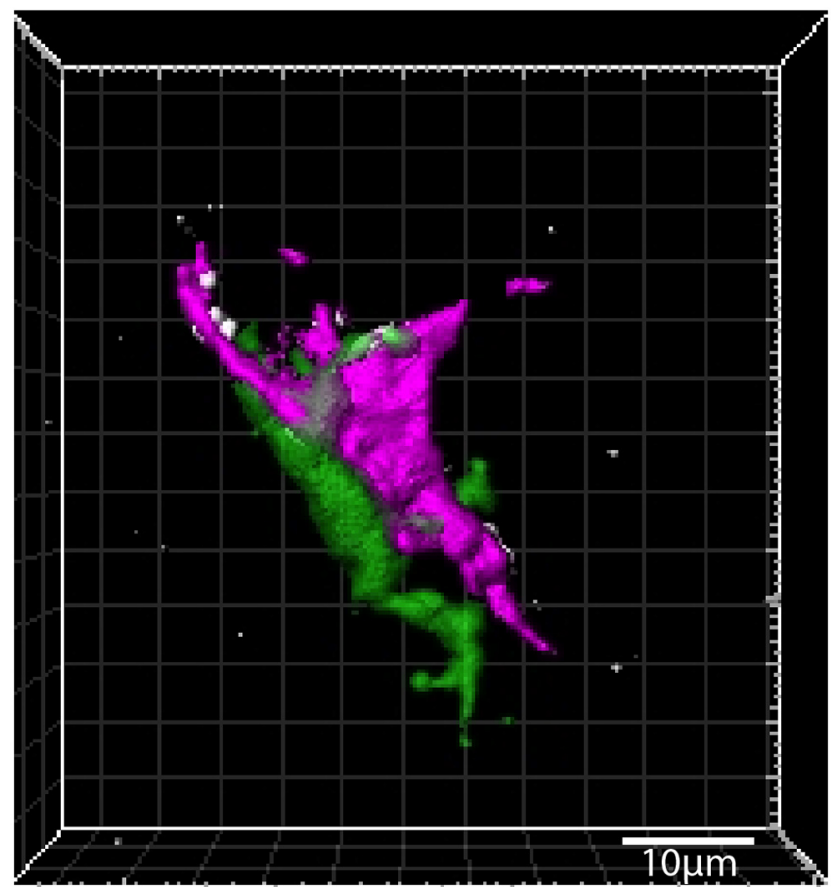

Movie 8. Juxtavascular microglia associate with the cortical vasculature in areas lacking full astrocytic endfoot coverage in the P5 frontal cortex. 3D reconstruction and surface rendering of juxtavascular microglia (green, EGFP) associated with blood vessels (magenta, antiPDGFR $\beta$ ) in areas void of astrocytic endfoot labeling (gray, anti-AQP4) in the frontal cortex at P5. [View online]

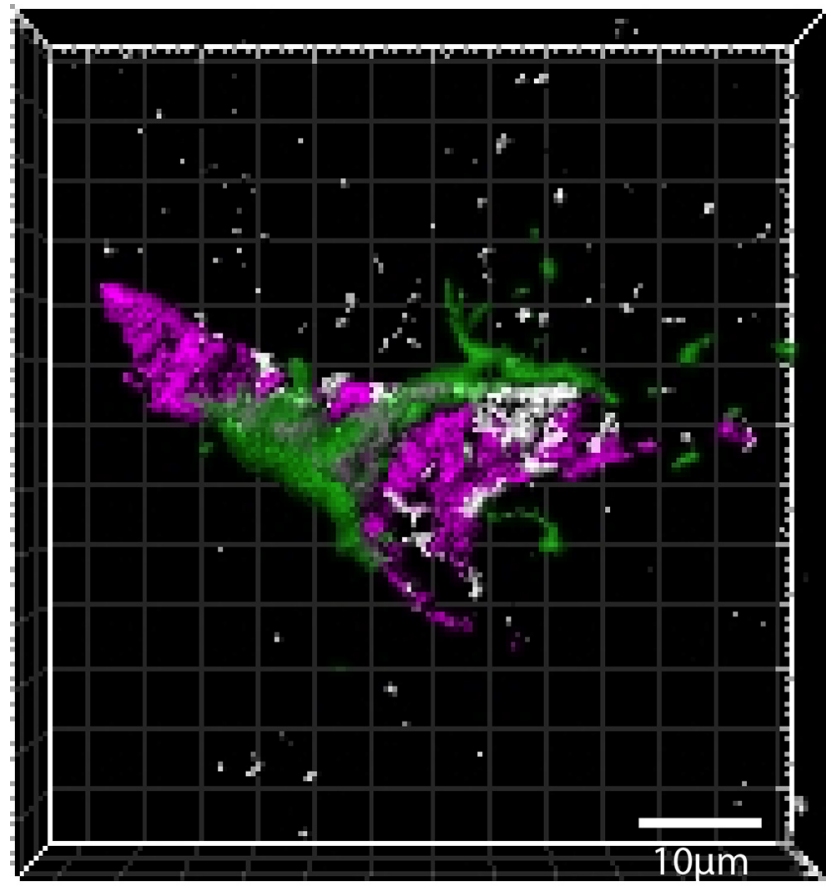

Movie 9. Juxtavascular microglia associate with the cortical vasculature in areas lacking full astrocytic endfoot coverage in the P7 frontal cortex. 3D reconstruction and surface rendering of juxtavascular microglia (green, EGFP) associated with blood vessels (magenta, antiPDGFR $\beta$ ) in areas void of astrocytic endfoot labeling (gray, anti-AQP4) in the frontal cortex at P7. [View online]

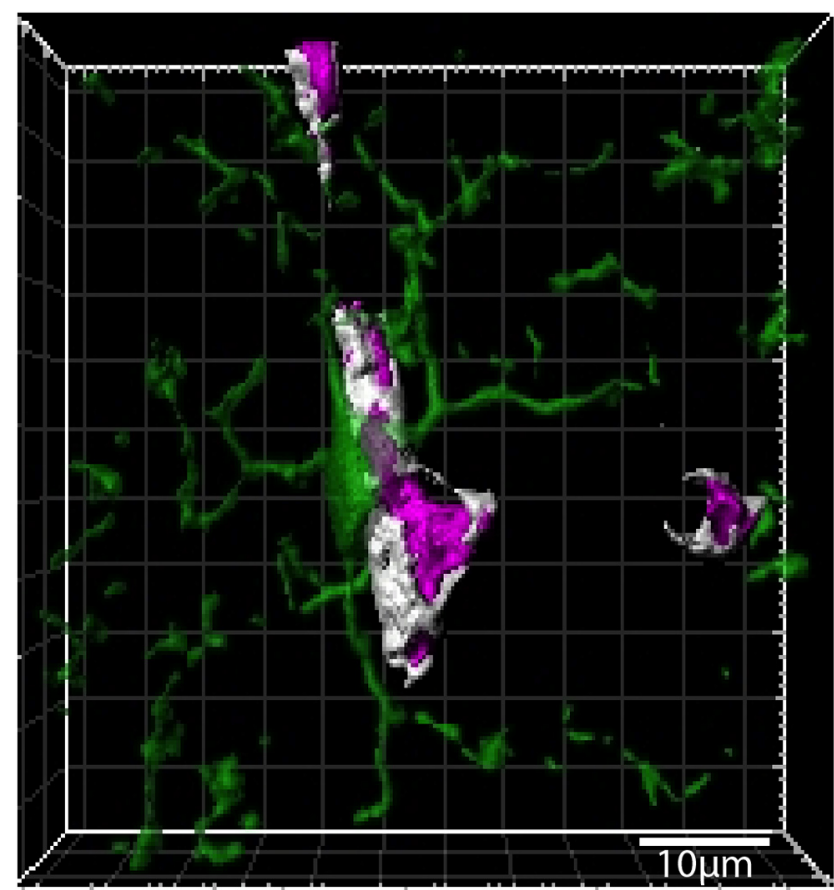

Movie 10. Juxtavascular microglia associate with the cortical vasculature in areas lacking full astrocytic endfoot coverage in the P28 frontal cortex. 3D reconstruction and surface rendering of juxtavascular microglia (green, EGFP) associated with blood vessels (magenta, anti$\operatorname{PDGFR} \beta$ ) in areas void of astrocytic endfoot labeling (gray, anti-AQP4) in the frontal cortex at P28. [View online]

are yet to be identified, which will be key to determining the relative importance of microglia-vascular interactions for microglial colonization, brain development, and long-term CNS function.

\section{Microglia-astrocyte interactions at the NVU interface}

Another interesting direction is to determine the role of astrocyte endfeet in regulating microglia-vascular interactions. Astrocytes are born and begin wrapping their processes to form endfeet along blood vessels during the first postnatal week (Daneman et al., 2010). By adulthood, astrocyte endfeet ensheath $60-95 \%$ of the vasculature (Mathiisen et al., 2010; Korogod et al., 2015). Here, we demonstrate that juxtavascular microglia in the postnatal cortex represent a large percentage of total microglia and are migratory along the vasculature. Juxtavascular microglia migration decreases as astrocyte endfeet develop and ensheath the vasculature. In addition, we showed that microglia associate with vessels at all ages in areas lacking full astrocytic endfoot coverage. EM revealed contact between juxtavascular microglia and the vascular basal lamina. These data raise the intriguing possibility that the basal lamina provides an adhesive substrate for microglial association and migration, which becomes restricted on astrocyte endfoot arrival. Astrocyte endfeet may, therefore, exclude microglia from contacting the basal lamina and associating with the vasculature. Another possibility is that microglia in the postnatal brain repel astrocyte endfeet, but this repellent signal later decreases as the animal matures so that astrocyte endfeet can wrap the vessels. Analysis of astrocyte endfoot-juxtavascular microglia interactions along blood vessels will be important going forward.

\section{Possible functions for juxtavascular microglia in the healthy} CNS

Are juxtavascular microglia a unique subpopulation of microglial cells that perform distinct functions at the NVU? Evidence in the 

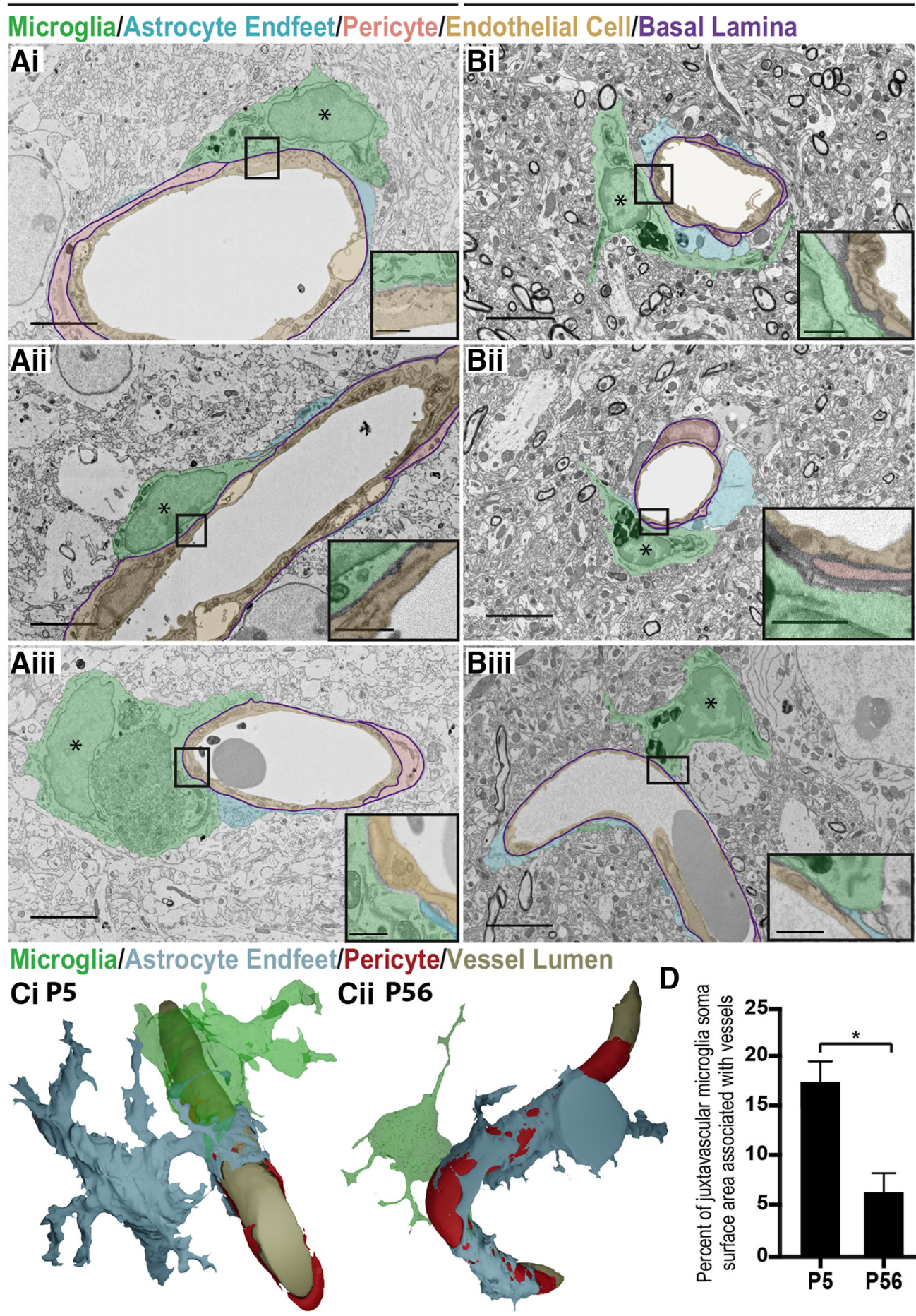

Figure 7. Ultrastructural analysis by EM reveals that juxtavascular microglia directly contact the basal lamina of the vasculature. Ai-Biii, EM of juxtavascular microglia (green pseudocoloring) contacting the basal lamina (purple line) of a blood vessel in an area void of astrocyte endfeet (blue pseudocoloring) in the P5 (Ai-Aiii, left column) and P56 (Bi-Biii, right column) frontal cortex. Pink pseudocoloring denotes pericytes and yellow pseudocoloring dentoes endothelial cells. Asterisks denote microglia nuclei. Scale bar, $5 \mu \mathrm{m}$. The black box denotes the magnified inset in the bottom right corner where microglia (green pseudocoloring) directly contact the basal lamina (unlabeled in the inset) and only partially contacts the astrocyte endfoot (blue pseudocoloring). Scale bar, $1 \mu \mathrm{m}$. Ci, Cii, 3D reconstruction of serial EM of P5 juxtavascular microglia (green pseudocoloring) in Aiii (Ci) and P56 juxtavascular microglia in Biii (Cii) contacting a blood vessel in an area void of astrocyte endfeet (blue pseudocoloring; Movies 11, 12). Red and tan pseudocoloring denotes a pericyte and vessel lumen, respectively. D, Quantification of the percentage of juxtavascular microglia contacting the basal lamina in single-optical plane images. Unpaired student's $t$ test; $n=29$ cells (P5) and $n=11$ cells (P56); ${ }^{*} p=0.0155$. All error bars represent \pm SEM.

literature suggests that microglia play important roles in regulating the vasculature, but it is unclear whether these functions are specific to juxtavascular microglia. For example, in the embryonic brain, microglia are often localized to vascular junction points, and the depletion of all microglia is associated with a decrease in vascular complexity (Fantin et al., 2010). Similar findings have been identified in the developing retina (Checchin et al., 2006; Rymo et al., 2011; Dudiki et al., 2020). Our data demonstrating that microglia are localized to the vasculature before the arrival of the astrocyte endfeet could place microglia in a 


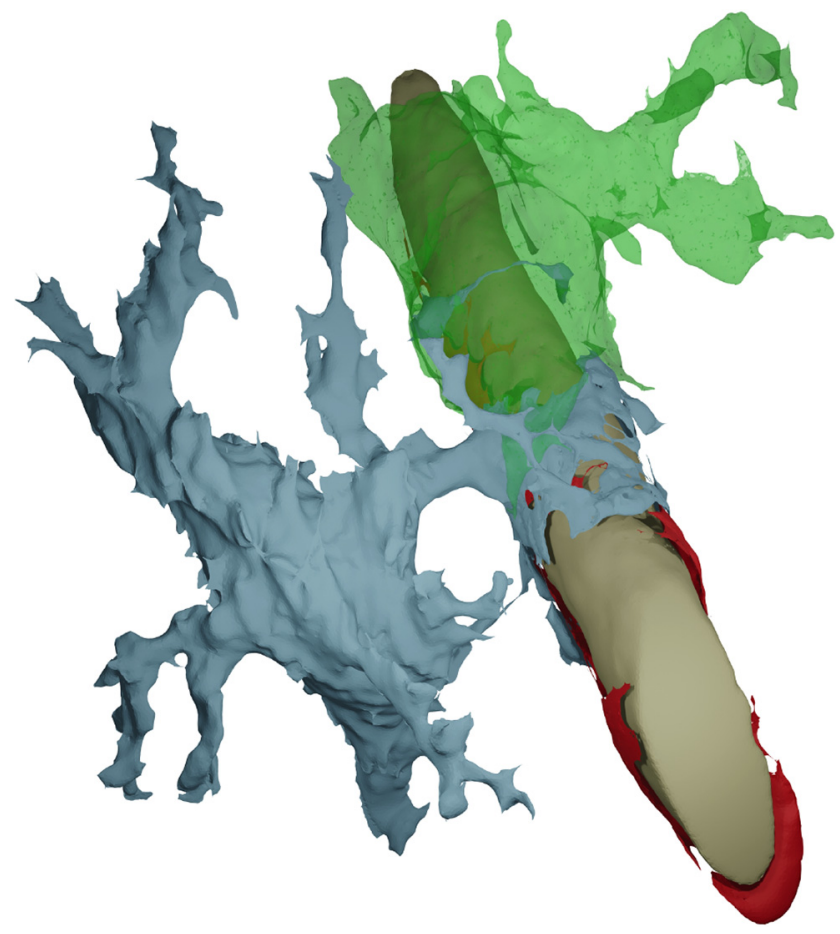

Movie 11. Serial EM 3D reconstruction of a juxtavascular microglia in the early postnatal cortex. 3D reconstruction of serial EM of juxtavascular microglia (green) contacting a blood vessel in an area void of astrocyte end feet (blue) in the P5 frontal cortex. Red and tan pseudocoloring denotes a pericyte and vessel lumen, respectively. [View online]

position to regulate fine-scale remodeling of the vasculature throughout the brain and/or help to maintain the BBB before astrocyte endfoot arrival. Arguing against the latter, microglia depletion during development does not appear to induce changes in BBB integrity in the postnatal brain (Parkhurst et al., 2013; Elmore et al., 2014). These data are in contrast to the inflamed adult CNS, where microglia regulate $\mathrm{BBB}$ integrity (Stankovic et al., 2016; Zhao et al., 2018). One of the most recent studies shows that during systemic inflammation, parenchymal microglia migrate to the vasculature and help to maintain the BBB at acute stages (Haruwaka et al., 2019). However, with sustained inflammation, microglia phagocytose astrocyte endfeet and facilitate BBB breakdown. In the absence of inflammation, it remains unknown what functions juxtavascular microglia may perform. In the adult, this could be a simple consequence of tiling with no specific functional implications. Arguing against this, we found that juxtavascular microglia are less evenly distributed and closer to their nearest neighbor microglia compared with vascular-unassociated microglia within the cortex. Also, our in vivo live-imaging data demonstrating microglia in the adult brain are stationary for nearly 2 months opens up the possibility that these cells could reside in a vascular niche. Although still speculative one possible role could be to serve as immunesurveillant "first responders." It will be important going forward to determine whether these juxtavascular microglia in the adult have unique functions.

\section{Microglia-vascular interactions: implications for CNS disease}

Our findings have important implications for neurologic diseases associated with the injured or aged CNS where there is enhanced microglial association with the vasculature, such as in stroke, brain tumors, multiple sclerosis, and Alzheimer's disease

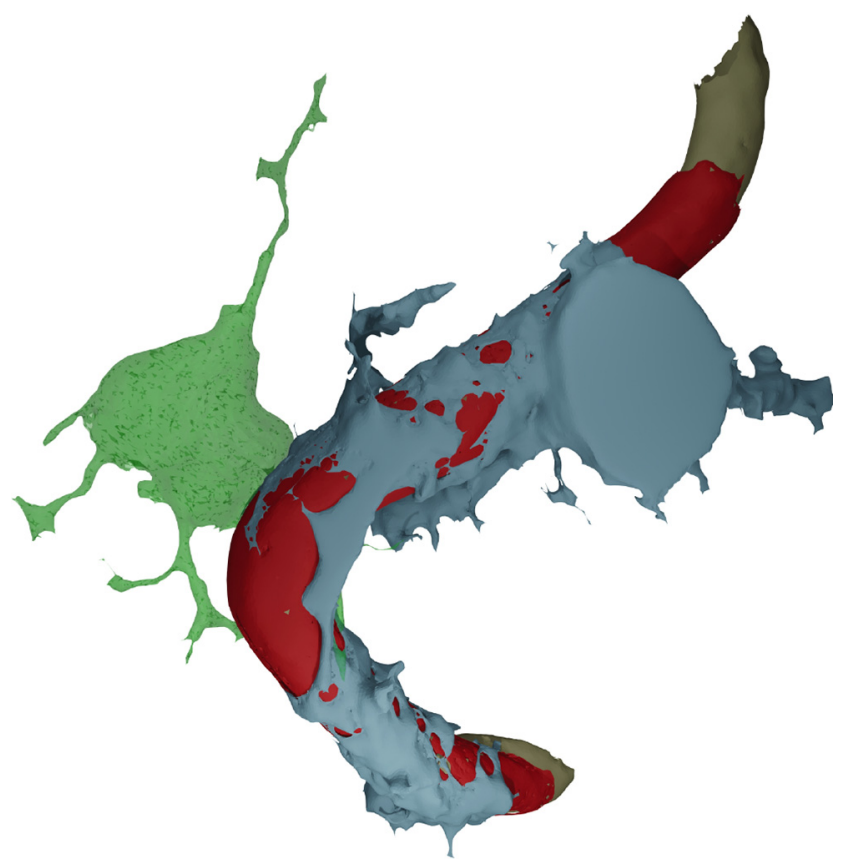

Movie 12. Serial EM 3D reconstruction of a juxtavascular microglia in the P56 cortex. 3D reconstruction of serial EM of juxtavascular microglia (green) contacting a blood vessel lacking full astrocyte endfoot (blue) coverage in the P56 frontal cortex. Red and tan pseudocoloring denotes a pericyte and vessel lumen, respectively. [View online]

(Stankovic et al., 2016; Zhao et al., 2018). This enhanced association can lead to further breakdown of the BBB, infiltration of peripheral immune cells into the CNS, and possibly angiogenesis, in the case of brain tumors (Stankovic et al., 2016; Zhao et al., 2018; Haruwaka et al., 2019). Therefore, understanding precisely when and where microglia interact with the vasculature in the healthy brain may lead to therapeutic strategies to reduce vascular pathology and facilitate recovery. One intriguing possibility is that these sites of juxtavascular microglia association with vessels, which lack astrocyte endfeet, are more vulnerable to BBB breakdown and infiltration of peripheral immune cells. In addition to neurodegenerative disorders, our findings may also have important implications for neurodevelopmental disorders such as autism spectrum disorders (ASDs). For example, microgliavascular interactions may be important for the timing of microglial colonization to synapse-dense brain regions where they regulate synapse maturation and pruning during critical windows in development (Tremblay et al., 2010; Paolicelli et al., 2011; Hoshiko et al., 2012; Schafer et al., 2012; Gunner et al., 2019). If these interactions are disrupted, the timing of synapse development and, ultimately, neural circuit function may be altered. This is supported by our data from $\mathrm{C} \times 3 \mathrm{crl}^{-/-}$mice showing delays in microglial association with the vessels, which is concomitant with known delays in microglial recruitment to developing synapses and delays in synapse maturation in these mice (Paolicelli et al., 2011; Hoshiko et al., 2012; Zhan et al., 2014). Long term, $C x 3 \mathrm{crl}^{-/-}$mice have phenotypes associated with ASD, including decreased functional brain connectivity, deficits in social interactions, and increased repetitive behaviors (Zhan et al., 2014). However, a better understanding of how vascular interactions affect microglial colonization and extending these analyses of microglia-vascular interactions into the ASD human brain will be necessary.

Together, our work sheds new light on an understudied population of microglia: juxtavascular microglia. This work lays the 
foundation for identifying new molecular mechanisms underlying microglia-vascular interactions, identifying mechanistic underpinnings of microglia-astrocyte cross talk at the level of the NVU, and furthering our understanding of juxtavascular microglia function in CNS homeostasis. With the vascular interface emerging as an important aspect of many neurologic conditions, this study also lays the critical groundwork to study how this microglial population may be important in a wide range of CNS diseases.

\section{References}

Abbott NJ, Rönnbäck L, Hansson E (2006) Astrocyte-endothelial interactions at the blood-brain barrier. Nat Rev Neurosci 7:41-53.

Abdel-Haq R, Schlachetzki JCM, Glass CK, Mazmanian SK (2019) Microbiome-microglia connections via the gut-brain axis. J Exp Med 216:41-59.

Adams RA, Bauer J, Flick MJ, Sikorski SL, Nuriel T, Lassmann H, Degen JL, Akassoglou K (2007) The fibrin-derived $\gamma$ 377-395 peptide inhibits microglia activation and suppresses relapsing paralysis in central nervous system autoimmune disease. J Exp Med 204:571-582.

Alliot F, Godin I, Pessac B (1999) Microglia derive from progenitors, originating from the yolk sac, and which proliferate in the brain. Brain Res Dev Brain Res 117:145-152.

Armulik A, Genové G, Mäe M, Nisancioglu MH, Wallgard E, Niaudet C, He L, Norlin J, Lindblom P, Strittmatter K, Johansson BR, Betsholtz C (2010) Pericytes regulate the blood-brain barrier. nature 468:557-561.

Arnò B, Grassivaro F, Rossi C, Bergamaschi A, Castiglioni V, Furlan R, Greter M, Favaro R, Comi G, Becher B, Martino G, Muzio L (2014) Neural progenitor cells orchestrate microglia migration and positioning into the developing cortex. Nat Commun 5:5611-5613.

Asano SM, Gao R, Wassie AT, Tillberg PW, Chen F, Boyden ES (2018) Expansion microscopy: protocols for imaging proteins and RNA in cells and tissues. Curr Protoc Cell Biol 80:e56.

Ashwell K (1991) The distribution of microglia and cell death in the fetal rat forebrain. Brain Res Dev Brain Res 58:1-12.

Bauer HC, Bauer H, Lametschwandtner A, Amberger A, Ruiz P, Steiner M (1993) Neovascularization and the appearance of morphological characteristics of the blood-brain barrier in the embryonic mouse central nervous system. Brain Res Dev Brain Res 75:269-278.

Bayraktar OA, Fuentealba LC, Alvarez-buylla A, Rowitch DH (2014) Astrocyte development and heterogeneity. Cold Spring Harb Perspect Biol 7:a020362.

Berger DR, Seung HS, Lichtman JW (2018) VAST (volume annotation and segmentation tool): efficient manual and semi-automatic labeling of large 3D image stacks. Front Neural Circuits 12:88.

Blender Foundation (2018) Blender-a 3D modelling and rendering package. Amsterdam: Blender Foundation.

Bovetti S, Hsieh Y-C, Bovolin P, Perroteau I, Kazunori T, Puche AC (2007) Blood vessels form a scaffold for neuroblast migration in the adult olfactory bulb. J Neurosci 27:5976-5980.

Brown LS, Foster CG, Courtney J-m, King NE, Howells DW, Sutherland BA, Johanna J, Sutherland BA (2019) Pericytes and neurovascular function in the healthy and diseased brain. Front Cell Neurosci 13:282.

Butovsky O, Jedrychowski MP, Moore CS, Cialic R, Lanser AJ, Gabriely G, Koeglsperger T, Dake B, Wu PM, Doykan CE, Fanek Z, Liu L, Chen Z, Rothstein JD, Ransohoff RM, Gygi SP, Antel JP, Weiner HL (2014) Identification of a unique TGF- $\beta$ dependent molecular and functional signature in microglia. Nat Neurosci 17:131-143.

Cardona A, Saalfeld S, Schindelin J, Arganda-Carreras I, Preibisch S, Longair M, Tomancak P, Hartenstein V, Douglas RJ (2012) TrakEM2 software for neural circuit reconstruction. PLoS One 7:e38011.

Checchin D, Sennlaub F, Levavasseur E, Leduc M, Chemtob S (2006) Potential role of microglia in retinal blood vessel formation. Invest Ophthalmol Vis Sci 47:3595-3602.

Daneman R (2012) The blood-brain barrier in health and disease. Ann Neurol 72:648-672.

Daneman R, Zhou L, Kebede AA, Barres BA (2010) Pericytes are required for blood-brain barrier integrity during embryogenesis. Nature 468:562566.
Davalos D, Grutzendler J, Yang G, Kim JV, Zuo Y, Jung S, Littman DR, Dustin ML, Gan W-B (2005) ATP mediates rapid microglial response to local brain injury in vivo. Nat Neurosci 8:752-758.

Davalos D, Kyu Ryu J, Merlini M, Baeten KM, Le Moan N, Petersen MA, Deerinck TJ, Smirnoff DS, Bedard C, Hakozaki H, Gonias Murray S, Ling JB, Lassmann H, Degen JL, Ellisman MH, Akassoglou K (2012) Fibrinogen-induced perivascular microglial clustering is required for the development of axonal damage in neuroinflammation. Nat Commun 3:1227.

Dudiki T, Meller J, Mahajan G, Liu H, Zhevlakova I, Ste S, Witherow C, Podrez E, Kothapalli CR, Byzova TV (2020) Microglia control vascular architecture via a TGF $\beta 1$ dependent paracrine mechanism linked to tissue mechanics. Nat Commun 11:986.

Elmore MRP, Najafi AR, Koike MA, Dagher NN, Spangenberg EE, Rice RA, Kitazawa M, Matusow B, Nguyen H, West BL, Green KN (2014) Colonystimulating factor 1 receptor signaling is necessary for microglia viability, unmasking a microglia progenitor cell in the adult brain. Neuron 82:380397.

Fantin A, Vieira JM, Gestri G, Denti L, Schwarz Q, Prykhozhij S, Peri F, Wilson SW, Ruhrberg C (2010) Tissue macrophages act as cellular chaperones for vascular anastomosis downstream of VEGF-mediated endothelial tip cell induction. Blood 116:829-841.

Frost JL, Schafer DP (2016) Microglia: architects of the developing nervous system. Trends Cell Biol 26:587-597.

Fujioka T, Kaneko N, Sawamoto K (2019) Blood vessels as a scaffold for neuronal migration. Neurochem Int 126:69-73.

Ginhoux F, Greter M, Leboeuf M, Nandi S, See P, Gokhan S, Mehler MF, Conway SJ, Ng LG, Stanley ER, Samokhvalov IM, Merad M (2010) Fate mapping analysis reveals that adult microglia derive from primitive macrophages. Science 330:841-845.

Goldey GJ, Roumis DK, Glickfeld LL, Kerlin AM, Clay Reid R, Bonin V, Schafer DP, Andermann ML (2014) Removable cranial windows for long-term imaging in awake mice. Nat Protoc 9:2515-2538.

Grant RI, Hartmann DA, Underly RG, Berthiaume A-A, Bhat NR, Shih AY (2019) Organizational hierarchy and structural diversity of microvascular pericytes in adult mouse cortex. J Cereb Blood Flow Metab 39:411-425.

Grossmann R, Stence N, Carr J, Fuller L, Waite M, Dailey ME (2002) Juxtavascular microglia migrate along brain microvessels following activation during early postnatal development. Glia 37:229-240.

Gunner G, Cheadle L, Johnson KM, Ayata P, Badimon A, Mondo E, Nagy MA, Liu L, Bemiller SM, Kim K-W, Lira SA, Lamb BT, Tapper AR, Ransohoff RM, Greenberg ME, Schaefer A, Schafer DP (2019) Sensory lesioning induces microglial synapse elimination via ADAM10 and fractalkine signaling. Nat Neurosci 22:1075-1088.

Hammond TR, Robinton D, Stevens B (2018) Microglia and the brain: complementary partners in development and disease. Annu Rev Cell Dev Biol 34:523-544.

Hanamsagar R, Bilbo SD (2017) Environment matters: microglia function and dysfunction in a changing world. Curr Opin Neurobiol 47:146-155.

Haruwaka K, Ikegami A, Tachibana Y, Ohno N, Konishi H, Hashimoto A, Matsumoto M, Kato D, Ono R, Kiyama H, Moorhouse AJ, Nabekura J, Wake H (2019) Dual microglia effects on blood brain barrier permeability induced by systemic inflammation. Nat Commun 10:5816.

Hoshiko M, Arnoux I, Avignone E, Yamamoto N, Audinat E (2012) Deficiency of the microglial receptor CX3CR1 impairs postnatal functional development of thalamocortical synapses in the barrel cortex. J Neurosci 32:15106-15111.

Kimelberg HK, Nedergaard M (2010) Functions of astrocytes and their potential as therapeutic targets. Neurotherapeutics 7:338-353.

Korogod N, Petersen CCH, Knott GW (2015) Ultrastructural analysis of adult mouse neocortex comparing aldehyde perfusion with cryo fixation. Elife 4:e05793.

Kubota Y, Takubo K, Shimizu T, Ohno H, Kishi K, Shibuya M, Saya H, Suda $\mathrm{T}$ (2009) M-CSF inhibition selectively targets pathological angiogenesis and lymphangiogenesis. J Exp Med 206:1089-1102.

Kubota Y, Sohn J, Hatada S, Schurr M, Straehle J, Gour A, Neujahr R, Miki T, Mikula S, Kawaguchi Y (2018) A carbon nanotube tape for serial-section electron microscopy of brain ultrastructure. Nat Commun 9:437433.

Lebovitz Y, Ringel-scaia VM, Allen IC, Theus HM (2018) Emerging developments in microbiome and microglia research: implications for neurodevelopmental disorders. Front Immunol 9:1993. 
Macvicar BA, Newman EA (2015) Astrocyte regulation of blood flow in the brain. Cold Spring Harb Perspect Biol 7:a020388.

Mastorakos P, Mcgavern D (2019) The anatomy and immunology of vasculature in the central nervous system. Sci Immunol 4:eaav0492.

Mathiisen TM, Lehre KP, Danbolt NC, Petter Ottersen OLE (2010) The perivascular astroglial sheath provides a complete covering of the brain microvessels: an electron microscopic 3D reconstruction. Glia 58:10941103.

McConnell HL, Kersch CN, Woltjer RL, Neuwelt EA (2017) The translational significance of the neurovascular unit. J Biol Chem 292:762-770.

Monier A, Adle-Biassette H, Delezoide A-L, Evrard P, Gressens P, Verney C (2007) Entry and distribution of microglial cells in human embryonic and fetal cerebral cortex. J Neuropathol Exp Neurol 66:372-382.

Navascués J, Calvente R, Marín-Teva JL, Cuadros MA (2000) Entry, dispersion and differentiation of microglia in the developing central nervous system. An Acad Bras Cienc 72:91-102.

Nikodemova M, Kimyon RS, De I, Small AL, Collier LS, Watters JJ (2015) Microglial numbers attain adult levels after undergoing a rapid decrease in cell number in the third postnatal week. J Neuroimmunol 278:280288.

Nimmerjahn A, Kirchhoff F, Helmchen F (2005) Resting microglial cells are highly dynamic surveillants of brain parenchyma in vivo. Science 308:1314-1319.

Paolicelli RC, Bolasco G, Pagani F, Maggi L, Scianni M, Panzanelli P, Giustetto M, Ferreira TA, Guiducci E, Dumas L, Ragozzino D, Gross CT (2011) Synaptic pruning by microglia is necessary for normal brain development. Science 333:1456-1458.

Parkhurst CN, Yang G, Ninan I, Savas JN, Yates JR, Lafaille JJ, Hempstead BL, Littman DR, Gan WB (2013) Microglia promote learning-dependent synapse formation through brain-derived neurotrophic factor. Cell 155:1596-1609.

Parslow AC, Cardona A, Bryson-richardson RJ (2014) Sample drift correction following 4D confocal time-lapse imaging. J Vis Exp (86):e51086.

Perry VH, Hume DA, Gordon S (1985) Immunohistochemical localization of macrophages and microglia in the adult and developing mouse brain. Neuroscience 15:313-326.

Rothhammer V, Borucki DM, Tjon EC, Takenaka MC, Chao C-C, ArduraFabregat A, de Lima KA, Gutiérrez-Vázquez C, Hewson P, Staszewski O, Blain M, Healy L, Neziraj T, Borio M, Wheeler M, Dragin LL, Laplaud DA, Antel J, Alvarez JI, Prinz M, et al. (2018) Microglial control of astrocytes in response to microbial metabolites. Nature 557:724-728.

Rymo SF, Gerhardt H, Wolfhagen Sand F, Lang R, Uv A, Betsholtz C (2011) A two-way communication between microglial cells and angiogenic sprouts regulates angiogenesis in aortic ring cultures. PLoS One 6: e15846.

Saili KS, Zurlinden TJ, Schwab AJ, Silvin A, Baker NC, Hunter ES, Ginhoux F, Knudsen TB (2017) Blood-brain barrier development: systems modeling and predictive toxicology. Birth Defects Res 109:1680-1710.

Schafer DP, Lehrman EK, Kautzman AG, Koyama R, Mardinly AR, Yamasaki R, Ransohoff RM, Greenberg ME, Barres BA, Stevens B (2012) Microglia sculpt postnatal neural circuits in an activity and complementdependent manner. Neuron 74:691-705.

Schiweck J, Eickholt BJ, Murk K (2018) Important shapeshifter: mechanisms allowing astrocytes to respond to the changing nervous system during development, injury and disease. Front Cell Neurosci 12:2611-2617.
Smolders SM-T, Swinnen N, Kessels S, Arnauts K, Smolders S, Le Bras B, Rigo J-M, Legendre P, Brône B (2017) Age-specific function of A5 $\beta 1$ integrin in microglial migration during early colonization of the developing mouse cortex. Glia 65:1072-1088.

Sorokin SP, Hoyt RF, Blunt DG, McNelly NA (1992) Macrophage development: II. Early ontogeny of macrophage populations in brain, liver, and lungs of rat embryos as revealed by a lectin marker. Anat Rec 232:527550 .

Stankovic N, Dudvarski M, Teodorczyk R, Ploen (2016) Microglia-blood vessel interactions: a double-edged sword in brain pathologies. Acta Neuropathol 131:347-363.

Swinnen N, Smolders S, Avila A, Notelaers K, Paesen R, Ameloot M, Brône B, Legendre P, Rigo JM (2013) Complex invasion pattern of the cerebral cortex by microglial cells during development of the mouse embryo. Glia 61:150-163.

Tapia JC, Kasthuri N, Hayworth K, Schalek R, Lichtman JW, Smith SJ, Buchanan J (2012) High contrast en bloc staining of neuronal tissue for field emission scanning electron microscopy. Nat Protoc 7:193-206.

Tinevez J-Y, Perry N, Schindelin J, Hoopes GM, Reynolds GD, Laplantine E, Bednarek SY, Shorte SL, Eliceiri KW (2017) TrackMate: an open and extensible platform for single-particle tracking. Methods 115:80-90.

Tremblay M-E, Lowery RL, Majewska AK (2010) Microglial interactions with synapses are modulated by visual experience. PLoS Biol 8:e1000527.

Tsai H-H, Niu J, Munji R, Davalos D, Chang J, Zhang H, Tien A-C, Kuo CJ, Chan JR, Daneman R, Fancy SPJ (2016) Oligodendrocyte precursors migrate along vasculature in the developing nervous system. Science 351:379-384.

Ueno M, Yamashita T (2014) Bidirectional tuning of microglia in the developing brain: from neurogenesis to neural circuit formation. Curr Opin Neurobiol 27:8-15.

Welker C, Woolsey TA (1974) Structure of layer IV in the somatosensory neocortex of the rat: description and comparison with the mouse. J Comp Neurol 158:437-453.

Whitman MC, Fan W, Rela L, Rodriguez-gil DJ, Greer CA (2009) Blood vessels form a migratory scaffold in the rostral migratory stream. J Comp Neurol 516:94-104

Woolsey TA, Van der Loos H (1970) The structural organization of layer IV in the somatosensory region (S I) of mouse cerebral cortex. Brain Res 17:205-242.

Yamanishi E, Takahashi M, Saga Y, Osumi N (2012) Penetration and differentiation of cephalic neural crest-derived cells in the developing mouse telencephalon. Dev Growth Differ 54:785-800.

Zeisel A, Munoz-Manchado AB, Codeluppi S, Lonnerberg P, La Manno G, Jureus A, Marques S, Munguba H, He L, Betsholtz C, Rolny C, CasteloBranco G, Hjerling-Leffler J, Linnarsson S (2015) Cell types in the moues cortex and hippocampus revealed by single-cell RNA-Seq. Science 347:1138-1143.

Zhan Y, Paolicelli RC, Sforazzini F, Weinhard L, Bolasco G, Pagani F, Vyssotski AL, Bifone A, Gozzi A, Ragozzino D, Gross CT (2014) Deficient neuron-microglia signaling results in impaired functional brain connectivity and social behavior. Nat Neurosci 17:400-406.

Zhao X, Eyo UB, Murugan M, Wu L-j (2018) Microglial interactions with the neurovascular system in physiology and pathology. Dev Neurobiol 78:604-617.

Zlokovic BV (2008) The blood-brain barrier in health and chronic neurodegenerative disorders. Neuron 57:178-201. 